\title{
Measuring magnetic fields from water masers in the synchrotron protostellar jet in $\mathrm{W} 3\left(\mathrm{H}_{2} \mathrm{O}\right)$
}

\author{
C. Goddi ${ }^{1,2}$, G. Surcis ${ }^{3,4}$, L. Moscadelli ${ }^{5}$, H. Imai ${ }^{6}$, W. H. T. Vlemmings ${ }^{7}$, H. J. van Langevelde ${ }^{3,8}$, and A. Sanna ${ }^{9}$ \\ ${ }^{1}$ Department of Astrophysics/IMAPP, Radboud University, PO Box 9010, 6500 GL Nijmegen, The Netherlands \\ e-mail: c.goddi@astro.ru.nl \\ 2 ALLEGRO/Leiden Observatory, Leiden University, PO Box 9513, 2300 RA Leiden, The Netherlands \\ 3 Joint Institute for VLBI in Europe, Postbus 2, 79990 AA Dwingeloo, The Netherlands \\ 4 INAF-Osservatorio Astronomico di Cagliari via della Scienza 5, 09047 Selargius, Italy \\ 5 INAF, Osservatorio Astrofisico di Arcetri, Largo E. Fermi 5, 50125 Firenze, Italy \\ 6 Department of Physics and Astronomy, Kagoshima University, 1-21-35 Korimoto, 890-0065 Kagoshima, Japan \\ 7 Chalmers University of Technology, Onsala Space Observatory, 43992 Onsala, Sweden \\ 8 Sterrewacht Leiden, Leiden University, PO Box 9513, 2300 RA Leiden, The Netherlands \\ 9 Max-Planck-Institut für Radioastronomie, Auf dem Hügel 69, 53121 Bonn, Germany
}

Received 15 July 2016 / Accepted 8 August 2016

\begin{abstract}
Context. Magnetic fields are invoked to launch, drive, and shape jets in both low- and high-mass protostars, but observational data on the spatial scales required to assess their role in the protostellar mass-loss process is still scarce.

Aims. The Turner-Welch (TW) object in the W3(OH) high-mass star-forming complex drives a synchrotron jet, which is quite exceptional for a high-mass protostar, and is associated with a strongly polarized $\mathrm{H}_{2} \mathrm{O}$ maser source, $\mathrm{W} 3\left(\mathrm{H}_{2} \mathrm{O}\right)$, making it an optimal target to investigate the role of magnetic fields on the innermost scales of protostellar disk-jet systems.

Methods. We report full polarimetric VLBA observations of $\mathrm{H}_{2} \mathrm{O}$ masers towards $\mathrm{W} 3\left(\mathrm{H}_{2} \mathrm{O}\right)$. Their linearly polarized emission provides clues on the orientation of the local magnetic field (on the plane of the sky), while the measurement of the Zeeman splitting provides its strength (along the line-of-sight). The linear scales probed by $\mathrm{H}_{2} \mathrm{O}$ masers are tens to hundreds of $\mathrm{AU}\left(\mathrm{at}\right.$ the W3( $\left.\mathrm{H}_{2} \mathrm{O}\right)$ distance, $\sim 2 \mathrm{kpc}$ ), inaccessible to other star-formation tracers.

Results. We identified a total of 148 individual maser features and we measured their physical properties. Out of 148, we measured linear polarization in 34 features, with a fractional percentage varying in the range $0.9-42 \%$, making $\mathrm{W} 3\left(\mathrm{H}_{2} \mathrm{O}\right)$ the highest-polarized $\mathrm{H}_{2} \mathrm{O}$ maser source observed with VLBI known in the Galaxy. The $\mathrm{H}_{2} \mathrm{O}$ masers trace a bipolar, biconical outflow at the center of the synchrotron jet. Although on scales of a few thousand AU the magnetic field inferred from the masers is on average orientated along the flow axis, on smaller scales (10s to 100s of AU), we have revealed a misalignment between the magnetic field and the velocity vectors, which arises from the compression of the field component along the shock front. We also detected circularly polarized emission toward ten maser features, with a fractional percentage varying in the range $0.2-1.6 \%$. In the gas shocked by the synchrotron jet, we estimate a total field strength in the range $\sim 100-300 \mathrm{mG}$ (at densities of $10^{9} \mathrm{~cm}^{-3}$ ). We conclude that fields of this order of magnitude are expected if the observed polarized water masers emerge behind magnetically supported shocks which, propagating in the $\mathrm{W} 3\left(\mathrm{H}_{2} \mathrm{O}\right)$ hot core (with an initial density of order of $10^{7} \mathrm{~cm}^{-3}$ ), compress and enhance the field component perpendicular to the shock velocity (with an initial field strength of a few $\mathrm{mG}$ ). We constrain the magnetic field strength in the pre-shock circumstellar gas (which is dominated by the component parallel to the flow motion) to at least $10-20 \mathrm{mG}$ (at densities of $10^{7} \mathrm{~cm}^{-3}$ ), consistent with previous estimates from a synchrotron jet model and dust polarization measurements.

Conclusions. In $\mathrm{W} 3\left(\mathrm{H}_{2} \mathrm{O}\right)$, the magnetic field would evolve from having a dominant component parallel to the outflow velocity in the pre-shock gas, with field strengths of the order of a few tens of $\mathrm{mG}$, to being mainly dominated by the perpendicular component of order of a few hundred of $\mathrm{mG}$ in the post-shock gas where the $\mathrm{H}_{2} \mathrm{O}$ masers are excited. The general implication is that in the undisturbed (i.e., not-shocked) circumstellar gas, the flow velocities would follow closely the magnetic field lines, while in the shocked gas the magnetic field would be reconfigured to be parallel to the shock front.
\end{abstract}

Key words. stars: formation - masers - instrumentation: interferometers - techniques: polarimetric - galaxies: star formation ISM: magnetic fields

\section{Introduction}

Protostellar jets from both low-mass and high-mass young stellar objects (YSOs) are known to be ionized, primarily through shocks, during early stages of their evolution (Anglada et al. 1998; Hofner et al. 2011). In the case of high-mass YSOs, the $\mathrm{H}$-ionizing luminosity increases dramatically when the YSO reaches the main sequence, resulting in prominent ionized winds and/or compact HII regions (Hoare et al. 2007). Therefore, thermal (bremsstrahlung) emission is often associated with (high-mass) YSOs, and observations of radio continuum as well as recombination lines can be naturally used to study the ionized component of (high-mass) protostar outflows.

Besides (thermal) free-free emission due to shock-induced ionization, low-mass YSOs like T Tauri stars are known to emit also (non-thermal) gyrosynchrotron emission from mildly relativistic electrons gyrating in magnetic fields (Andre 1996). Nonthermal emission in radio jets has been also observed in some 
massive protostars, for example $\mathrm{W} 3\left(\mathrm{H}_{2} \mathrm{O}\right)$ (Reid et al. 1995), HH 80-81 (Carrasco-González et al. 2010), IRAS 18182-1433 (Hofner et al. 2011), and G16.59-0.05 (Moscadelli et al. 2013). These findings have been interpreted in terms of synchrotron emission from relativistic electrons accelerated in strong shocks, but overall synchrotron jets appear to be quite rare. However, a recent study by Moscadelli et al. (2016) suggests that nonthermal continuum emission could be common in high-mass protostellar jets, after all. Studying the magnetic field in synchrotron jets provides a great opportunity to understand its role in the protostellar mass-loss process and, more in general, in high-mass star formation (HMSF).

The Turner-Welch (TW) object (Turner \& Welch 1984) is a luminous hot-core $\left(L \sim 10^{4} L_{\odot}\right)$ about $6^{\prime \prime}$ or $10^{4} \mathrm{AU}$ east of the archetypal ultracompact (UC) HII region W3(OH), at a distance of $2.04 \mathrm{kpc}$ (Hachisuka et al. 2006). With respect to the neighboring $\mathrm{W} 3(\mathrm{OH})$, it shows weak $\mathrm{OH}$ masers and no UC-HII region, indicating an earlier evolutionary stage. The dense molecular clump is forming a protobinary (or a multiple) system, as inferred from the structure in the $\mathrm{mm}$ dust emission (Wyrowski et al. 1999; Zapata et al. 2011). Remarkably, the most massive member of the multiple (labelled A; Wyrowski et al. 1999) is driving a synchrotron jet (Reid et al. 1995; Wilner et al. 1999), along the east-west (E-W) direction, which is also the direction along which the multiple is forming. Besides the synchrotron jet, the TW-object powers strong $\mathrm{H}_{2} \mathrm{O}$ masers, therefore the core is also indicated as $\mathrm{W} 3\left(\mathrm{H}_{2} \mathrm{O}\right)$. Proper motions measurements of these water masers revealed a bipolar outflow centered at the synchrotron jet, expanding at about $20 \mathrm{~km} \mathrm{~s}^{-1}$ (Alcolea et al. 1993; Hachisuka et al. 2006).

One interesting property of molecular masers is that, besides being superb kinematic probes, they can be polarized. With a detailed theory of maser polarization propagation, polarimetric observations can yield the strength of the magnetic field along the line-of-sight (l.o.s.) from circular polarization (Zeeman effect) and the 2D (or even the 3D) field structure from linear polarization (see Vlemmings 2012, for a review). And indeed, a number of water maser sources have been studied with polarization observations, and all showed that the linear polarization vectors of individual water maser features are generally aligned with each other, indicating that the measured polarization truly probes the magnetic field in the maser region (Imai et al. 2003; Vlemmings et al. 2006; Surcis et al. 2011a,b, 2014).

In this paper, we present full polarimetric observations of $\mathrm{H}_{2} \mathrm{O}$ masers in $\mathrm{W} 3\left(\mathrm{H}_{2} \mathrm{O}\right)$, with the main goal of measuring for the first time the magnetic field strength and structure along the synchrotron jet driven by a high-mass protostar. The general question we want to address is whether there is a coupling between the gas motion and the magnetic field, that is if the gas motion locally follows the magnetic field or vice versa. In order to address this question, we need to probe the small scales where jets and winds are expected to be launched and collimated by massive protostars (a few tens to hundreds of AU; see for example Matthews et al. 2010; Greenhill et al. 2013). This requires very long baseline interferometry (VLBI) measurements.

\section{Observations and data reduction}

We observed the massive star-forming region $\mathrm{W} 3(\mathrm{OH})$ in the $6_{16}-5_{23}$ transition of $\mathrm{H}_{2} \mathrm{O}$ (rest frequency: $22.23508 \mathrm{GHz}$ ) with the NRAO ${ }^{1}$ Very Long Baseline Array (VLBA) on August 18th 2006. The observations were conducted in full polarization spectral mode using one single baseband filter of $2 \mathrm{MHz}$, which covered a total velocity range of $\approx 27 \mathrm{~km} \mathrm{~s}^{-1}$. We performed three correlation passes in total. The first one had 128 channels with a spectral resolution of $15.6 \mathrm{kHz}\left(0.2 \mathrm{~km} \mathrm{~s}^{-1}\right)$ and included all 4 polarization combinations: RR, LL, RL, LR. This pass allowed to produce images in all Stokes parameters and was used in the analysis to measure the linear polarization of individual maser features. The other two passes had higher spectral resolution $\left(1.95 \mathrm{kHz}\right.$ or $0.03 \mathrm{~km} \mathrm{~s}^{-1}$ ) and 1024 channels, and contained only the circular polarization combinations: RR and LL, respectively. These extra two passes were required to measure Zeeman splitting of individual $\mathrm{H}_{2} \mathrm{O}$ maser lines. Including the overheads, the total observation time was $12 \mathrm{~h}$.

The data were calibrated and imaged using the Astronomical Image Processing Software package (AIPS). The calibration of the bandpass, delay, rate, and phase was performed on the calibrators 0420-014 and DA193. The fringe-fitting and the self-calibration were performed on the brightest maser feature at $V_{\mathrm{LSR}}=-48.6 \mathrm{~km} \mathrm{~s}^{-1}$ (Table D.1), on the dataset with $0.2 \mathrm{~km} \mathrm{~s}^{-1}$ velocity resolution. In order to calibrate the polarization, we first removed instrumental effects, by estimating the D-terms for each antenna. For this purpose, we used 0420-014, which is a linearly polarized source (2\%; Marscher et al. 2002), covering a large variation $\left(>90^{\circ}\right)$ of the parallactic angle during the observations. The solutions from fringe-fitting and D-terms were then transferred from the moderate to the high spectral resolution dataset. We did not correct for the R-L delay offset, which affects the polarization angle but in a negligible way with respect to its uncertainty.

Imaging and deconvolution of the two fully calibrated data sets were performed within the AIPS task IMAGR, using Briggs weighting with $\mathcal{R}=0$ and a pixel size of

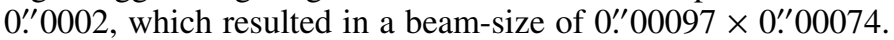
The phase-center position was: $\alpha(\mathrm{J} 2000)=02^{\mathrm{h}} 27^{\mathrm{m}} 04.836$, $\delta(\mathrm{J} 2000)=+61^{\circ} 52^{\prime} 24.61^{\prime \prime}$, corresponding to the TW-object. Since the water maser distribution extends E-W across more than $3^{\prime \prime}$ (Hachisuka et al. 2006), we simultaneously imaged three fields of $8192 \times 8192$ pixels or 1 . $^{\prime \prime} 6 \times 1$ I. $^{\prime \prime} 6$ each around the TW object, sufficient to cover the total area with known water maser emission. A fourth field was imaged approximately $7^{\prime \prime}$ to the west of the TW-object, at the position of the HII region $\mathrm{W} 3(\mathrm{OH})$, but no water maser emission was detected. For each field, we created data cubes of Stokes $I\left(\mathrm{rms}=7.3 \mathrm{mJy} \mathrm{beam}^{-1}\right)$, $Q\left(\mathrm{rms}=6.5 \mathrm{mJy} \mathrm{beam}^{-1}\right)$ and $U\left(\mathrm{rms}=6.5 \mathrm{mJy} \mathrm{beam}^{-1}\right)$ for the modest spectral resolution dataset, and Stokes $I$ and $V$ $\left(\mathrm{rms}=10 \mathrm{mJy}\right.$ beam $\left.^{-1}\right)$ for the high spectral resolution dataset. The $Q$ and $U$ cubes were combined to produce cubes of polarized intensity $\left(P O L I=\sqrt{Q^{2}+U^{2}}\right)$ and polarization angle $(P O L A=1 / 2 \times \operatorname{atan}(U / Q))$. We calibrated the linear polarization angles of individual $\mathrm{H}_{2} \mathrm{O}$ masers by rotating the linear polarization angle measured for 0420-014 from our VLBA data to the one measured in a POLCAL VLA observation ${ }^{2}$ carried out 43 days before our VLBA observations: $\chi_{0420-014}=+66^{\circ} .91$. The formal errors on the POLA values for individual spectral channels are due to thermal noise: $\sigma_{\text {POLA }}=0.5 \sigma_{P} / P \times 180^{\circ} / \pi$ (Wardle \& Kronberg 1974), where $P$ and $\sigma_{P}$ are the polarization intensity and corresponding rms error, respectively. The $I$

\footnotetext{
1 The National Radio Astronomy Observatory (NRAO) is a facility of the National Science Foundation operated under cooperative agreement by Associated Universities, Inc.

2 http://WwW.aoc.nrao.edu/ smyers/calibration/
} 
and $V$ cubes at high spectral resolution were used to evaluate the magnetic field strength along the line of sight, which is proportional to the circular polarization fraction $P_{\mathrm{V}}=\left(V_{\max }-\right.$ $\left.V_{\min }\right) / I_{\max }$. The analysis of the maser polarization data is described in Appendix A.

We did not observe in phase-referencing mode, therefore during self-calibration we lose the information on the absolute position. The positions of the identified $\mathrm{H}_{2} \mathrm{O}$ masers are relative to the strongest maser feature used for self-calibration at $-48.6 \mathrm{~km} \mathrm{~s}^{-1}$. In order to estimate the absolute position in our maps, we used the astrometric measurements by Hachisuka et al. (2006) (the procedure is described in Appendix B).

\section{Results}

We used the VLBA at $22 \mathrm{GHz}$ to perform full polarization observations of water masers toward $\mathrm{W} 3\left(\mathrm{H}_{2} \mathrm{O}\right)$. We identified a total of 148 individual maser features and we measured their physical properties, including positions, flux densities, 1.o.s. velocities $\left(V_{\mathrm{LSR}}\right)$, and (when polarized) their fraction of linear and circular polarizations, as well as the corresponding linear polarization angles and magnetic field strengths along the 1.o.s. (these parameters are listed in Table D.1). The methodology adopted to derive the physical properties of individual maser features is described in Appendix A.

The identified maser features have peak flux densities from $50 \mathrm{mJy}$ to about $2000 \mathrm{Jy}$, and $V_{\mathrm{LSR}}$ spanning from -60.6 to $-39.7 \mathrm{~km} \mathrm{~s}^{-1}$. Figure 1 shows positions and l.o.s. velocities of the $22 \mathrm{GHz}$ water maser features overplotted on the contour map of the dust continuum emission at $1.4 \mathrm{~mm}$, imaged with the PdBI by Wyrowski et al. (1999). The masers are distributed over an area of 2 ." $5 \times 0$.' 5 along E-W, and are clustered in five main groups, which we label with letters from $a$ to $e$. The l.o.s. velocities do not show any particularly ordered distribution, consistent with a flow in the plane of the sky (Reid et al. 1995). The $1.4 \mathrm{~mm}$ continuum is elongated E-W, across approximately $3^{\prime \prime}$, and comprises three dusty components, labelled A, B, and C from east to west (Wyrowski et al. 1999). The masers are loosely associated with these three peaks of dust emission with the majority of $\mathrm{H}_{2} \mathrm{O}$ masers detected towards the strongest dust continuum peak (A) and to the east from it, at the eastern "tip" of the elongated dusty core, where also the highest intensity maser features are observed.

Besides positions and 1.o.s. velocities, we also measured the position angle of the linear polarization vectors $(\chi)$ and the Zeeman splitting for single maser features. We describe these measurements in the next two subsections.

\subsection{Linear polarization: magnetic field orientation}

Out of the 148 features detected, we measured linear polarization in 34 maser features, with a fractional percentage varying in the range $P_{1}=0.9-42 \%$ (see Table D.1 and Appendix A). In Fig. 1, line segments indicate the linear polarization vectors of the maser features, whose length scales logarithmically with the polarization fraction, $P_{l}$. Remarkably, 13 features have $P_{1}>10 \%$, with 4 having $P_{1}>35 \%$, making $\mathrm{W} 3\left(\mathrm{H}_{2} \mathrm{O}\right)$ the highest-polarized $\mathrm{H}_{2} \mathrm{O}$ maser source on VLBI scales known in the Galaxy ${ }^{3}$. This is both a blessing and a curse, because on the

\footnotetext{
3 Before this work, the highest polarization fraction of water masers (26\%) measured with VLBI was found in W75N (Surcis et al. 2014). Besides VLBI, higher fractions of linear polarization were detected with
}

one hand such high fractions of polarization are obviously easier to measure (flux-wise), but on the other hand these masers are most probably saturated, and their polarized signal does not reflect the source magnetic field (see for example the discussion in Garay et al. 1989, in the case of the $\mathrm{H}_{2} \mathrm{O}$ maser flare in Orion $\mathrm{BN}-\mathrm{KL}$ ). In particular, this hampers the exact relation between the polarization vector and the magnetic field orientation. According the theory of polarized maser emission (Goldreich et al. 1973), the direction of the linear polarization vector is either parallel or perpendicular to the magnetic field (sky-projected) orientation, depending if the angle between the field orientation and the maser propagation direction (the so called Van Vleck angle, $\theta$ ) is less than or over $55^{\circ}$, respectively.

In order to assess weather $\theta$ is less or more than $55^{\circ}$, we used a two-steps approach. First, we used the full radiative transfer method (FRTM) code developed by Vlemmings (2006) and based on the model by Nedoluha \& Watson (1992), to determine $\theta$ (and associated errors) in each individual maser feature with linearly polarized emission (see Appendix A). We then considered the entire range of values allowed by the estimated errors, from $\theta-\Delta \theta$ to $\theta+\Delta \theta$. In cases where $\theta-\Delta \theta>55^{\circ}$, the magnetic field is undoubtedly perpendicular to the linear polarization vector, whereas for $\theta+\Delta \theta<55^{\circ}$ it is undoubtedly parallel. In cases where $\theta-\Delta \theta<55^{\circ}$ and $\theta+\Delta \theta>55^{\circ}$, the magnetic field is either perpendicular or parallel to the linear polarization vector. In those cases, we assumed that the magnetic field is more likely parallel if $\left|(\theta+\Delta \theta)-55^{\circ}\right|<\left|(\theta-\Delta \theta)-55^{\circ}\right|$ (otherwise more likely perpendicular). It turned out that most of the maser features with a relatively low (i.e., a few percent) fractional polarization fall in this last group, owing to larger negative error-bars with respect to positive error-bars (see Appendix A for an explanation).

As a second step, we plotted the magnetic field vectors according to their $\theta$ angles calculated in the first step (these include all the linearly polarized features displayed in Fig. 1). We started analyzing the richest maser cluster $a$, which displays a remarkably consistent orientation of the linear polarization vectors (Fig. 1, inset a). We noticed however that the presumed magnetic field orientations of adjacent masers changed by $\sim 90^{\circ}$ in several cases. In fact, one limitation in our analysis is that the FRTM code overestimates $\theta$ for saturated masers (Vlemmings 2006), which implies that the condition of perpendicularity is erroneously satisfied in some cases. Therefore, sudden changes of $90^{\circ}$ in magnetic field orientation probed by adjacent masers may indicate that saturation effects are at play. Notably, when excluding maser features with $P_{1} \geq 5 \%$, we found that the presumed magnetic field orientations of adjacent masers become consistent with one another (within the errors). We interpret this result in terms of an empirical threshold of linear polarization fraction $\left(P_{1}=5 \%\right)$ above which the water masers enter into the saturation regime, and therefore their polarized signal does not trace the magnetic field anymore. The goodness of this criterion is also demonstrated by the fact that it automatically excludes all the features in cluster $d$ (except one), which indeed show a number of properties ascribable to saturation: the highest intensity, including flaring features (up to $2000 \mathrm{Jy}$ ), among the highest values of brightness temperature, and an origin in a turbulent region interested by strong shocks. This may explain the exceptional high values of their linear polarization fraction $(>10 \%$; see Table D.1) and the random distribution of their linear polarization vectors (see inset $d$ in Fig. 1), indicating that the polarization does not reflect the magnetic field in the region.

single-dish measurements (e.g., Garay et al. 1989 registered a 60\% fraction in a $\mathrm{H}_{2} \mathrm{O}$ maser flare in Orion $\mathrm{BN}-\mathrm{KL}$ ) 

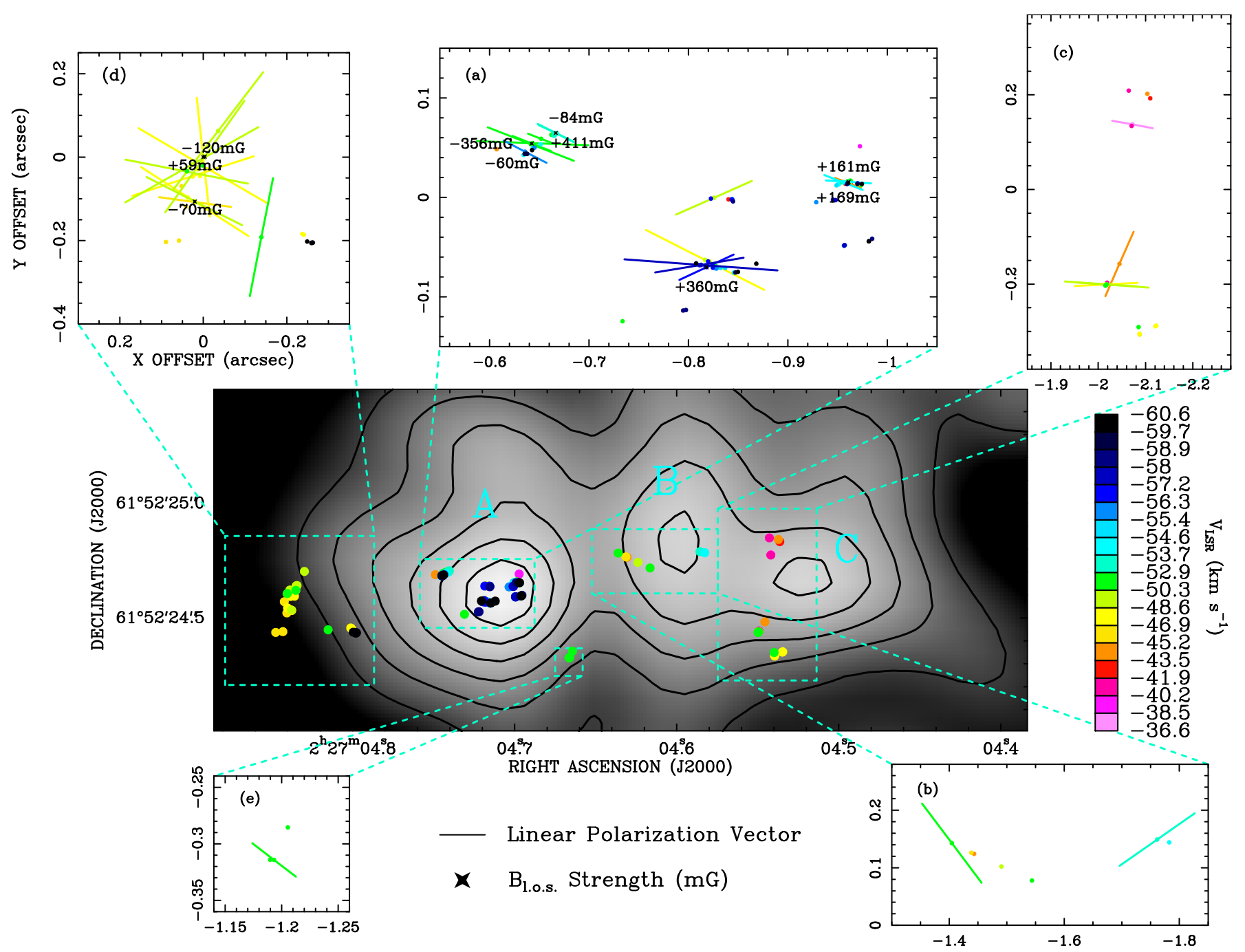

Fig. 1. Overlay of the water masers detected with the VLBA in $\mathrm{W} 3\left(\mathrm{H}_{2} \mathrm{O}\right)$ onto the $1.4 \mathrm{~mm}$ continuum emission mapped with the PdBI by Wyrowski et al. (1999) (gray scale and black contours). The circles show positions of the $\mathrm{H}_{2} \mathrm{O}$ masers, while the colors denote their l.o.s. velocity in $\mathrm{km} \mathrm{s}^{-1}$ (color scale on the right-hand side). The three $1.4 \mathrm{~mm}$ continuum peaks identified by Wyrowski et al. (1999) are labelled A, B, and C, from east to west. Contour levels correspond to steps of $24 \mathrm{mJy}_{\text {beam }}^{-1}$ (starting from $47 \mathrm{mJy} \mathrm{beam}^{-1}$ ). The insets show the linear polarization vectors of individual maser features in different clusters (from $a$ to $e$ ), where the length of the line segments scales logarithmically with the polarization fraction (in the range $P_{1}=0.9-42 \%$ ). We also report the magnetic field strengths (in $\mathrm{mG}$ ) along the 1.o.s. $\left(B_{1.0 . \text {. }}\right.$ ) in the maser features for which we measured the Zeeman splitting. The positions are relative to the reference maser feature used for data self-calibration (ID 018 in Table D.1).

In summary, in order to avoid contamination from saturated masers, in our analysis we adopted a conservative approach by excluding all maser features with $P_{1} \geq 5 \%$, which are either saturated or going towards a saturation state. A similar result was obtained in previous works by Vlemmings et al. (2006) and Surcis et al. (2011a), who also suggested that a high linear polarization fraction, $P_{l}>5 \%$, can only be produced when the maser is saturated.

Based on this analysis, we found that the magnetic field is parallel to the linear polarization vectors for most of the identified maser features with $P_{1}<5 \%$ (these have their $\theta$ angles in boldface in Table D.1). Figure 2 shows the resulting (skyprojected) magnetic field vectors for the maser features with $P_{1}<$ $5 \%$, overplotted on the $1.4 \mathrm{~mm}$ continuum map tracing the dust emission (same as in Fig. 1), as well as the $8.4 \mathrm{GHz}$ continuum imaged with the VLA (Wilner et al. 1999). Interestingly, the magnetic field inferred from the $\mathrm{H}_{2} \mathrm{O}$ masers is on average oriented along E-W, that is, along the $\mathrm{mm}$ dust and radio continuum emissions, suggesting a physical relation between them (see Sect. 4.1). The fact that the field orientations for neighboring maser features are mostly consistent with one another, increases our confidence in the physical relevance of the inferred magnetic field structure.

\subsection{Circular polarization: magnetic field strength}

Besides linear polarization, we also detected circularly polarized emission toward ten maser features, varying in the range $P_{\mathrm{V}}=0.2-1.6 \%$. Considering only masers with $P_{1}<5 \%$, that is, 037, 051, 063, 104, and 105 (all towards component A of the mm continuum), we obtain values of the magnetic field strength along the l.o.s., $B_{\text {l.o.s. }}$, of $(-60 \pm 11) \mathrm{mG},(-84 \pm 35)$ $\mathrm{mG},(+360 \pm 120) \mathrm{mG},(+169 \pm 34) \mathrm{mG}$, and $(+161 \pm 41) \mathrm{mG}$, respectively ${ }^{4}$.

\footnotetext{
4 A negative magnetic field strength indicates that the magnetic field is pointing toward the observer; positive away from the observer.
} 


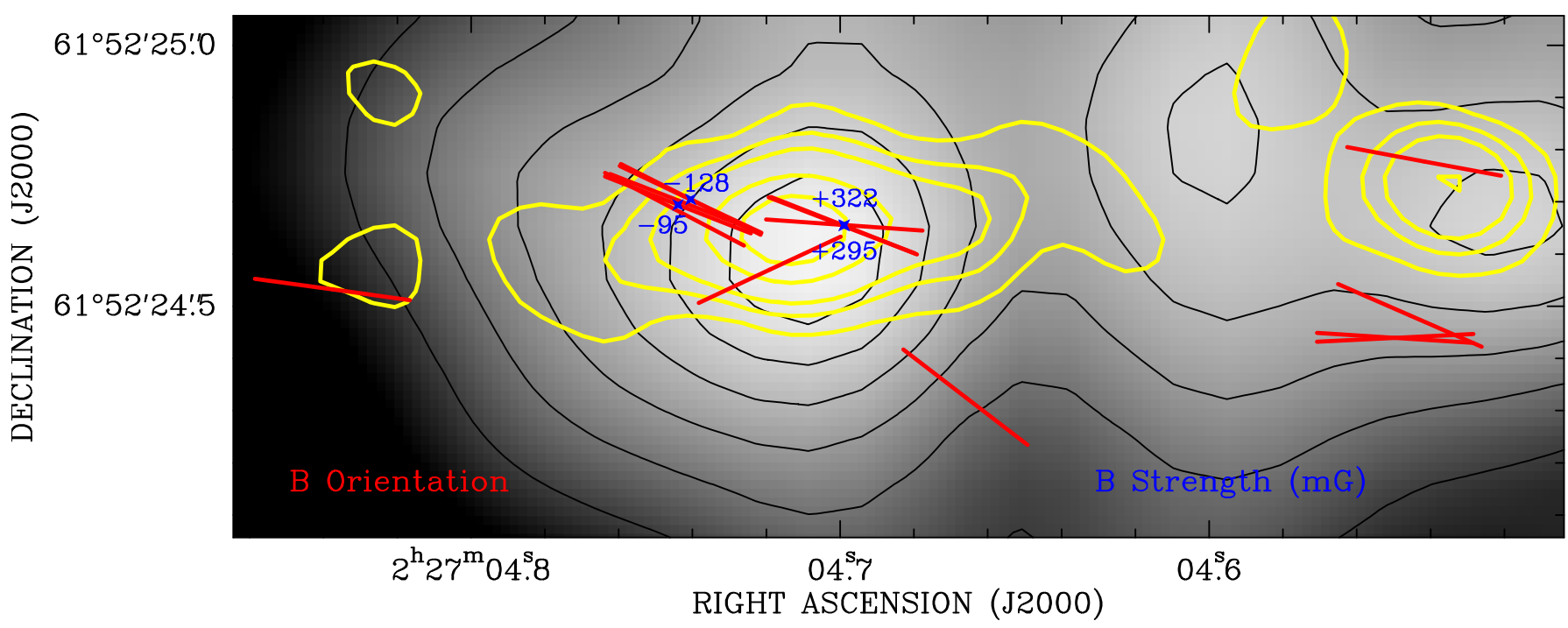

Fig. 2. Magnetic field orientation (in the plane of the sky) for 17 individual masers with $P_{l}<5 \%$ (red segment) and strength for four (nonsaturated) masers for which the Zeeman splitting was measured. The $8.4 \mathrm{GHz}$ emission imaged with the VLA (beamsize $\sim 0$.' 2 ) by Wilner et al. (1999) (yellow contours: corresponding to $0.02,0.06,0.1,0.2,0.3 \mathrm{mJy}_{\text {beam }}^{-1}$ ) is overplotted onto the $1.4 \mathrm{~mm}$ continuum emission mapped with the PdBI (beamsize 0.'5) by Wyrowski et al. (1999) (gray scale and black contours: same as in Fig. 1). The radio continuum shows a main central component, the synchrotron jet, and two (western and eastern) secondary components (see Sect. 4.1 for an interpretation).

Sarma et al. (2002) also conducted Zeeman measurements in the $22 \mathrm{GHz} \mathrm{H}_{2} \mathrm{O}$ masers with the VLA (beamsize $\sim 0$.' 1 ), yielding a field strength $B_{\text {l.o.s. }}=42 \pm 3 \mathrm{mG}$, much lower than the values we quote here. However, their measurements used only the strongest feature at $-49.1 \mathrm{~km} \mathrm{~s}^{-1}$, located at $\alpha(\mathrm{J} 2000)=$ $02^{\mathrm{h}} 27^{\mathrm{m}} 04.866, \delta(\mathrm{J} 2000)=+61^{\circ} 52^{\prime} 24.89^{\prime \prime}$, that is, in the cluster $d$ of $\mathrm{H}_{2} \mathrm{O}$ masers (Fig. 1, inset d), while our measurements of circular polarization include only cluster $a$. We also measured a Zeeman splitting for the same feature detected with the VLA (corresponding to maser ID 018 in Table D.1), yielding $B_{\text {l.o.s. }}=59 \pm 9 \mathrm{mG}$ : this is consistent with the value inferred by Sarma et al. $(2002)^{5}$. We did not however include this feature (nor any other strong maser feature in cluster $d$ ) in our analysis because it does not satisfy the condition that the masers should be unsaturated, and therefore cannot provide reliable estimates of the magnetic field strength. On the other hand, we fear that a measurement of the Zeeman splitting towards our cluster $a$ with the VLA may have been prevented by spatial blending of features with positive and negative components of $B_{\text {l.o.s. }}$ with similar strengths within the VLA beam (one example is provided by features 036 and 039).

The total amplitude of the magnetic field can be estimated with $B=B_{\text {l.o.s. }} / \cos (\theta)$, yielding $B_{037}=-95_{+45}^{-20} \mathrm{mG}, B_{051}=$ $-128_{+77}^{-57} \mathrm{mG}, B_{104}=+295_{-30}^{+214} \mathrm{mG}$, and $B_{105}=+322_{-37}^{+256} \mathrm{mG}^{6}$ (we excluded the maser component 063 because $\theta$ is $\sim 90^{\circ}$, and $B$ cannot be constrained). The locations of the maser features for which we measured the Zeeman splitting, along with the associated values of the magnetic field strengths, are plotted in Fig. 2 and reported in Table 1 .

It is perhaps worth noting that the amplitude of the magnetic field strength decreases with distance from the radio and mm continuum peaks in component $\mathrm{A}$, and has opposite signs on either side of the continuum peak (see Fig. 2 and Table 1).

\footnotetext{
The small discrepancy between the VLBI and VLA values may be due to blending with other features within the VLA beam (e.g., features 018 and 019), as also noticed by Sarma et al. (2002) based on the asymmetry in the maser line profile, which required multiple components for a satisfactorily Gaussian fit.
}

6 The errors in $B$ take into consideration the errors of both $B_{\text {l.o.s. }}$ and $\theta$
Table 1. Measurements of the magnetic field strength from $\mathrm{H}_{2} \mathrm{O}$ masers with Zeeman splitting in TW-A.

\begin{tabular}{ccccc}
\hline \hline $\begin{array}{c}\text { Maser } \\
\text { ID }\end{array}$ & $\begin{array}{c}\text { RA (J2000) } \\
(\mathrm{h}: \mathrm{m}: \mathrm{s})\end{array}$ & $\begin{array}{c}\text { Dec (J2000) } \\
\left({ }^{\circ}::^{\prime}\right)\end{array}$ & $\begin{array}{c}B \text { strength } \\
(\mathrm{mG})\end{array}$ & $\begin{array}{c}R^{a} \\
(\operatorname{arcsec})\end{array}$ \\
\hline 037 & $02: 27: 04.7444$ & $61: 52: 24.694$ & $-95_{++45}^{-20}$ & 0.347 \\
051 & $02: 27: 04.7409$ & $61: 52: 24.705$ & $-128_{+77}^{-57}$ & 0.314 \\
104 & $02: 27: 04.6994$ & $61: 52: 24.655$ & $+295_{-30}^{+214}$ & 0.110 \\
105 & $02: 27: 04.6993$ & $61: 52: 24.656$ & $+322_{-37}^{+256}$ & 0.111 \\
\hline
\end{tabular}

Notes. ${ }^{(a)}$ Sky-projected separation between the masers with Zeeman splitting measurements and the putative location of the TW-A protostar, estimated from the peak of the $8.4 \mathrm{GHz}$ continuum, $\alpha_{2000}=$ $02^{\mathrm{h}} 27^{\mathrm{m}} 04^{\mathrm{s}} .7103$ and $\delta_{2000}=+061^{\circ} 52^{\prime} 24^{\prime \prime} .650$.

In Sect. 4.1 we show that the continuum peak locates the protostellar position and the radio continuum emission comes from the protostellar jet. Therefore, this finding may indicate that the magnetic field decreases as a function of distance from the protostar along the jet axis, probing both the redshifted and blueshifted lobes of the molecular outflow.

\section{Discussion}

One relevant question in HMSF is the relation between the gas dynamics and the magnetic field in regulating mass-accretion and mass-loss. VLBI measurements of $\mathrm{H}_{2} \mathrm{O}$ masers can provide a detailed description of gas kinematics and magnetic field structure on scales from tens to hundreds AU, which are the smallest accessible scales in studies of HMSF, and have therefore the potential to address such an open question. W3 $\left(\mathrm{H}_{2} \mathrm{O}\right)$ contains the best known (archetypical) case of synchrotron jet driven by an embedded high-mass YSO and associated with $\mathrm{H}_{2} \mathrm{O}$ masers, and therefore provides a good target for investigating this issue. Alcolea et al. (1993), and later on Hachisuka et al. (2006), performed multi-epoch VLBI observations of $\mathrm{H}_{2} \mathrm{O}$ masers, thus probing the $3 \mathrm{D}$ velocity field of the molecular gas surrounding the synchrotron jet on linear scales of hundreds of $\mathrm{AU}$. 

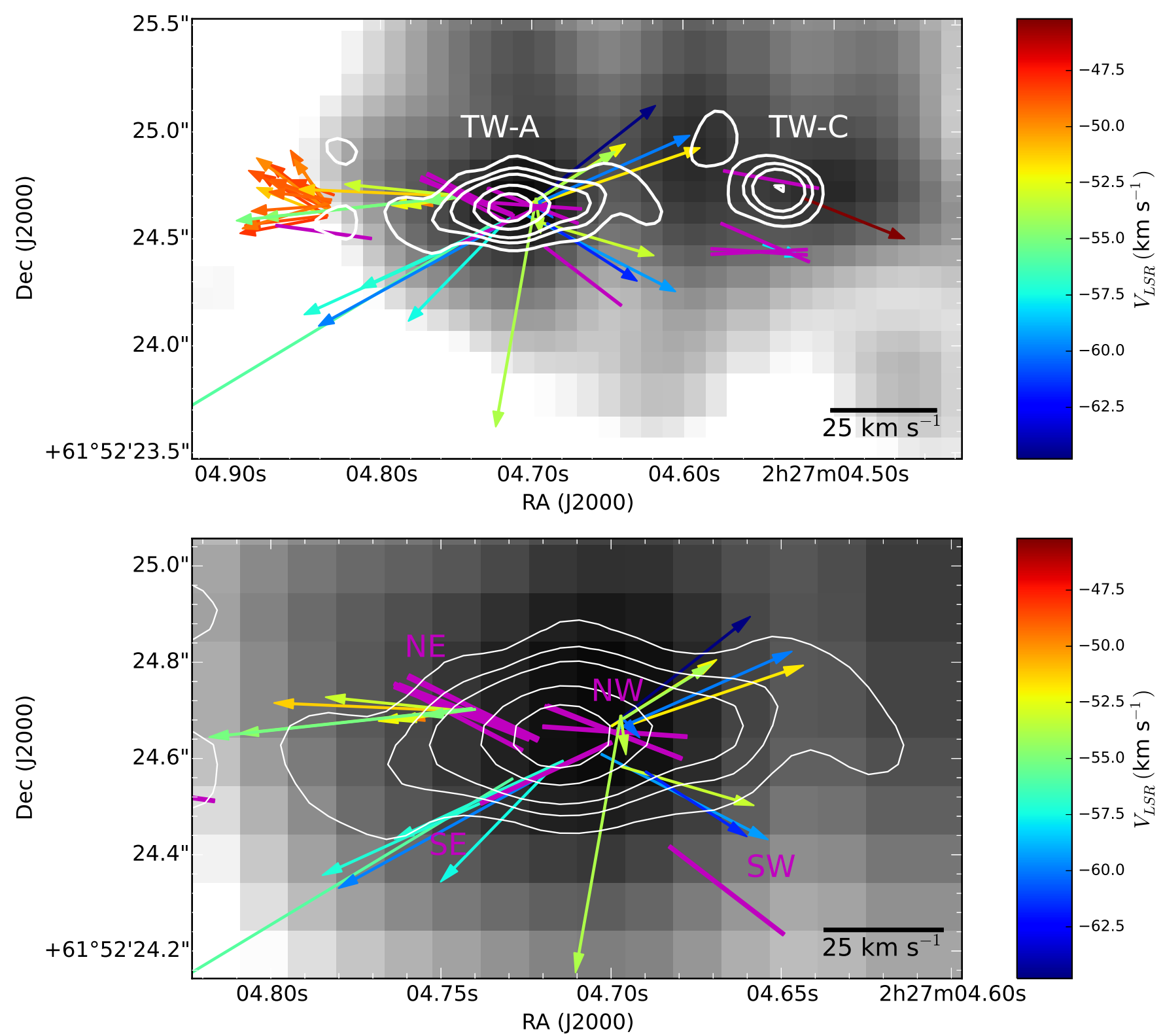

Fig. 3. Comparison between the magnetic field orientations of $\mathrm{H}_{2} \mathrm{O}$ masers (purple segments) measured in this work and proper motions of $\mathrm{H}_{2} \mathrm{O}$ masers (arrows) measured by Hachisuka et al. (2006). The $8.4 \mathrm{GHz}$ emission imaged with the VLA by Wilner et al. (1999) (white contours) is overplotted onto the $1.4 \mathrm{~mm}$ continuum emission mapped with the PdBI by Wyrowski et al. (1999) (gray scale). Colors denote 1.o.s. velocity (color scales on the right-hand side) and the scale for the proper motion amplitude is given in the lower right corner (both in $\mathrm{km} \mathrm{s}^{-1}$ ). Top panel: the outward proper motions of the masers to the east and west suggest the presence of a collimated bipolar molecular outflow along E-W (the axis of the synchrotron jet), whereas the proper motions of the masers closer to the radio continuum peak identify a wider-angle biconical outflow driven by the TW-A high-mass protostar. The western component identifies TW-C, the binary companion of TW-A (e.g., Wyrowski et al. 1999; Zapata et al. 2011). Bottom panel: expanded view towards the core of the synchrotron jet, showing more in details the relation between the $\mathrm{H}_{2} \mathrm{O}$ masers magnetic field orientations (purple segments) and proper motions (arrows). There are four main knots of masers, located towards NE, NW, SE, and SW, with respect to the radio continuum peak (labeled accordingly in the figure). The proper motions identify a biconical, bipolar molecular outflow driven by the TW-A high-mass protostar. We note the misalignment between magnetic field and velocity vectors, particularly in the NE and NW knots (see Sect. 4.2 for an explanation).

Our polarimetric measurements of $\mathrm{H}_{2} \mathrm{O}$ masers enabled us to infer the magnetic field strength and orientation on similar linear scales, thus providing the missing piece of information to help us assess the above-mentioned relation.

\subsection{Gas dynamics and magnetic field structure}

Figure 3 shows an overlay of the dust emission from the hot-core (grayscale; Wyrowski et al. 1999), synchrotron emission from the radio jet (white contours; Wilner et al. 1999), water maser proper motions (arrows) measured by Hachisuka et al. (2006), and the direction of magnetic field vectors as determined from our polarization measurements (purple segments). This overlay illustrates a number of physical properties of the system that we describe in detail below.

The first most notable element is the presence of multiple components in the radio continuum, as already noticed by (Wilner et al. 1999): a central strongest component, and two 
secondary peaks to the west (stronger) and to the east (fainter), respectively. The strongest component is centered at the $\mathrm{mm}$ dust continuum peak A (see also Fig. 1) and has an elongated morphology along E-W: this is the synchrotron jet discovered by Reid et al. (1995). Considered their alignment with the axis of the central jet, the secondary components were initially both interpreted as Herbig-Haro objects resulting from strong shocks produced as the fast protostellar jet impinges onto the dense ambient medium. While this may explain the (weaker) eastern component, an alternative interpretation has been suggested for the western (stronger) component, which is nearly coincident with the dust continuum peak $\mathrm{C}$, and shows a rising spectral in$\operatorname{dex}(\alpha=+0.9$ between 8 and $15 \mathrm{GHz}-$ Wilner et al. 1999), as expected from an ionized stellar wind and/or thermal radio jet. Wyrowski et al. (1999) used several $K$ components of the HNCO (10-9) transition to map the rotational temperature of the molecular gas in $\mathrm{W} 3\left(\mathrm{H}_{2} \mathrm{O}\right)$, and found evidence for two heating sources in the region (with $T_{k} \sim 200 \mathrm{~K}$ ), coincident with the dusty peaks $\mathrm{A}$ and $\mathrm{C}$. These findings are interpreted as evidence for a binary made of two high-mass (B-type) protostars embedded in the $\mathrm{W} 3\left(\mathrm{H}_{2} \mathrm{O}\right)$ hot molecular core (Zapata et al. 2011). In the remaining of this paper, we indicate these protostars as TWA and TW-C (these are labeled in the top panel of Fig. 3).

The second element worth discussing is the kinematics traced by $\mathrm{H}_{2} \mathrm{O}$ masers. They are mainly concentrated towards the three components of the radio continuum, along E-W, with the eastern and western maser clusters showing outward proper motions at about $20 \mathrm{~km} \mathrm{~s}^{-1}$. The natural interpretation is that the $\mathrm{H}_{2} \mathrm{O}$ masers probe the molecular component of the synchrotron jet (e.g., Hachisuka et al. 2006). In fact, a closer look at the measured proper motions in different clusters reveals additional features. First, there is a strong asymmetry between the eastern and western maser clusters. The eastern cluster contains the strongest, saturated masers (with peak flux densities up to about $2000 \mathrm{Jy}$ ), and shows a remarkably linear structure perpendicular to its motion (see also Fig. 1, inset d), reminiscent of a bowshock or a propagating exciting front (similar to what seen in other $\mathrm{H}_{2} \mathrm{O}$ maser sources: e.g., G24.78; Moscadelli et al. 2007, and Cepheus A; Torrelles et al. 2001): this structure can be a component of the same jet propagating from TW-A. On the other hand, the western component excites a much smaller number of (weaker) masers, which seem to move consistently towards south-west: these masers are most probably associated with the second YSO, TW-B. Finally, in the central cluster (labelled a in Fig. 1), the masers show a biconical expanding flow centered on the presumed location of the exciting high-mass protostar, TWA, with an opening angle of about $15-30^{\circ}$ (estimated from the proper motion position angles) and with somewhat higher velocities (up to $90 \mathrm{~km} \mathrm{~s}^{-1}$ ) with respect to the external components. This suggests that the outflow from the TW-A object has potentially two different components, a collimated synchrotron jet elongated E-W across a few thousand of AU and a somewhat wider-angle biconical molecular flow at its center (a similar outflow structure is observed in the high-mass Cepheus A HW2 system; Torrelles et al. 2011).

The third feature displayed in Fig. 3 (top panel), is the apparent consistency between gas kinematics and magnetic field orientation, at least on scales of order of one thousand AU or larger, comparable with the size of the dusty core. Indeed, the average magnetic field orientation inferred from the $\mathrm{H}_{2} \mathrm{O}$ masers is mainly E-W, that is, well aligned with the axis of the synchrotron jet as well as with the axis the molecular outflow (the latter can be defined by the average orientation of the maser proper motions). This is also apparently consistent with the dust polarized emission, mapped with the SMA at $0.9 \mathrm{~mm}$ (1.'5 beamsize; Chen et al. 2012), which shows a magnetic field aligned with the E-W elongation of the dusty core surrounding the TW-object(s). This finding is however surprising since the $\mathrm{mm}$ emission traces quiescent material in the dusty hot core, while $\mathrm{H}_{2} \mathrm{O}$ masers trace shocked material in the prostostellar jet. Therefore, the two probes are not expected to provide necessarily consistent pictures for the magnetic field.

The much higher angular resolution provided by the VLBI measurements, with respect to imaging of polarized emission from dust, enables us to take a much closer look at the relation between gas kinematics and magnetic field morphology. The bottom panel of Fig. 3 offers a zoomed view of the innermost parts of the synchrotron jet within 1500 AU from TW-A. The first notable feature is the presence of four different knots of masers, located towards NE, NW, SE, and SW with respect to the radio continuum peak, respectively (these knots are labeled accordingly in the figure). Their proper motions identify a clear biconical expanding flow (as already noticed). Since we also determined the magnetic field orientation for some of the polarized masers associated with these four different knots, we can directly compare it with the velocity vectors. One caveat is that, since the multi-epoch proper motion and the polarimetric experiments were conducted five to six years apart, a one-to-one comparison between magnetic field and proper motion orientations in individual maser features is not possible. Nevertheless, we can still compare their average relative orientations at different locations. We find that the angles representing the minimum differences between the average position angles of proper motions and magnetic fields in the plane of the sky are respectively: $6^{\circ}$ for SE, $14^{\circ}$ for $\mathrm{SW}, 30^{\circ}$ for $\mathrm{NE}$, and $54^{\circ}$ in the $\mathrm{NW}^{7}$. This finding indicates that, on scales of few hundreds AU, there is a significant misalignment between the orientations of the magnetic field and the velocity vectors. The origin of such a misalignment can be understood with an origin of water masers in fast shocks, as we detail in next section.

\subsection{Magnetically supported shocks}

Water masers are thought to emerge behind fast C- or J-type shocks (Hollenbach \& McKee 1979; Elitzur et al. 1989; Kaufman \& Neufeld 1996; Hollenbach et al. 2013). We show here that the magnetic field properties (strength and orientation) inferred from the observed polarized water maser emission are indeed consistent with an origin in (magnetically supported) shocks.

As a shock propagates in the ambient medium, it alters the initial magnetic field configuration in the circumstellar gas; in particular, the magnetic field component perpendicular to the shock velocity (and parallel to the shock front) is compressed and, therefore, enhanced with respect to the parallel component (which remains unaffected). As a consequence, we expect the magnetic fields probed by the $\mathrm{H}_{2} \mathrm{O}$ masers to be along the shock front $^{8}$ (see quantitative discussion in Sect. 4.2.1). This implies that the $\mathrm{H}_{2} \mathrm{O}$ masers provide the structure of the magnetic field in the post-shock gas, rather than in the quiescent circumstellar gas, and therefore they are a good probe of the shock morphology. Nevertheless, when the information on the measured

\footnotetext{
These are the values for the angle $\phi$ defined in Sect. 4.2.1 and Appendix C.

8 Observational biases also favor the same component because of maser path length, which will favor the compressed field perpendicular to the velocity and parallel to the shock.
} 
orientation and strength of the magnetic field (in the post-shock gas) is coupled with the knowledge of the maser velocities, we can still obtain constraints on the strength and geometry of the magnetic field in the pre-shock circumstellar gas (as we detail in orientation and strength 4.2.2).

\subsubsection{Geometry of the magnetic field in the post-shock gas}

After the shock passage, the component of the magnetic field parallel to the shock surface, and therefore perpendicular to the shock velocity, is expected to become dominant in the postshock gas. This implies a well defined prediction on the expected relative orientation between the gas motion and the magnetic field. In the assumption that the proper motions of water masers closely represent the shock velocity ${ }^{9}$, we would expect an angle of $90^{\circ}$ between the magnetic field and the proper motion orientations. However, as pointed out in Sect. 4.1, the observed angles between the sky-projected component of the magnetic field and the proper motions are significantly less than $90^{\circ}$ (see also the bottom panel of Fig. 3). A natural explanation for the apparent deviation from the expected perpendicularity between magnetic field and velocity vectors is conductible to $3 \mathrm{D}$ effects.

In Appendix $\mathrm{C}$, we show that the ratio between the components of the magnetic field parallel, $B_{\|}$, and perpendicular, $B_{\perp}$, to the proper motion orientation can be expressed as follows:

$B_{\perp} / B_{\|}=\sqrt{\cot ^{2}(\theta) / \cos ^{2}(\phi)+\tan ^{2}(\phi)}$

where $\theta$ is the angle between the magnetic field vector $\boldsymbol{B}$ and the line of sight (as already defined in Sect. 3.1), and $\phi$ is the angle between the sky-projected magnetic field and the proper motion orientation (whose measured values are given at the end of Sect. 4.1).

This ratio can provide a measure of the relative orientation of the magnetic field with respect to the proper motions. In a first approximation, if $B_{\perp}>$ or $\gg B_{\|}$, then the magnetic field can be considered most probably perpendicular to the proper motions. In order to have $B_{\perp}>B_{\|}, \phi$ should be large and $\theta$ should be small. Although we find $\phi \leq 30^{\circ}$ in three (out of four) of the knots with measured proper motion and magnetic field orientations (the NW knot has $\phi=54^{\circ}$; see end of Sect. 4.1), those masers present a large negative errorbars in the measured values of $\theta$ (see the boldface numbers reported in Col. 13 of Table D.1), allowing values as low as $15^{\circ} \leq \theta \leq 30^{\circ}$. As an example, using Eq. (1) and taking the maximum value in the range of $\theta=30^{\circ}$ and a minimum value of $\phi=10^{\circ}$, we obtain a minimum value of $B_{\perp} \approx 1.8 B_{\|}$, implying a lower limit of $\approx 60^{\circ}$ for the angle between the magnetic field orientation and the maser velocity (i.e., the shock velocity). On the other hand, taking a minimum allowed value of $\theta \approx 15^{\circ}$ and a maximum value of $\phi \approx 50^{\circ}$, it would imply a maximum ratio $B_{\perp} / B_{\|} \approx 6$, corresponding to an angle of $\approx 80^{\circ}$ between the magnetic field and the shock velocity.

This simple analysis shows that, the observed misalignment between the magnetic field and the velocity vectors in the plane of the sky, can be explained with an origin in magnetically supported shocks, where (the post-shock) magnetic field is expected to be perpendicular to the maser (and shock) velocity.

A caveat is that our analysis is based on measurements of magnetic fields and velocities that were not simultaneous, which prevented us to compare the two properties within individual

\footnotetext{
9 Since the proper motions dominate over the line-of-sight velocity, here we assume that the shocks responsible for the excitation of $\mathrm{H}_{2} \mathrm{O}$ masers move in the plane of the sky.
}

maser features. Therefore the observed relation between magnetic field and velocity vectors is confirmed on scales of the identified maser knots, that is several tens of AU. Future, simultaneous observations of water maser polarization and proper motions will allow us to verify if such a relation holds from the smallest scales of individual maser features to scales of the entire outflow. The power of simultaneous observations of polarized emission and proper motions has been recently demonstrated in the case of 6.7 GHz methanol masers by Sanna et al. (2015), who assessed the existence of a coupling between the magnetic field and the motion of the circumstellar gas. Unlike methanol, water masers are shock-excited, therefore establishing the same relation is less straightforward in the case of water masers. We discuss this aspect in Sect. 4.2.2.

\subsubsection{Constraints on the strength and geometry of the magnetic field in the pre-shock gas}

While $\mathrm{H}_{2} \mathrm{O}$ masers provide direct information on the (magnetic and kinematic) structure of the shock, polarization and proper motion measurements (possibly simultaneous) can be used together to get constraints on the magnetic field properties in the pre-shock gas as well.

In order to relate the magnetic field strength in the preand post-shock region, we can adopt the J-shock model of Hollenbach \& McKee (1979). In particular, for a magnetically supported shock, Hollenbach \& McKee (1979) showed that by equating the ram pressure of the pre-shock gas and the magnetic pressure of the post-shock gas, one obtains (their Eq. (2.34)):

$\left(\frac{B_{0 \perp}}{10^{-3} \mathrm{mG}}\right) \sim 77\left(\frac{v_{\mathrm{s}}}{100 \mathrm{kms}^{-1}}\right) \frac{n_{0}^{3 / 2}}{n_{\mathrm{s}}}$

where $B_{0 \perp}$ is the component of the field compressed by the shock (perpendicular to its propagating direction), $v_{\mathrm{s}}$ is the shock velocity, $n_{0}$ and $n_{\mathrm{s}}$ are the pre-shock and post-shock gas densities ${ }^{10}$, respectively. By assuming a pre-shock density of $10^{7} \mathrm{~cm}^{-3}$ (Wyrowski et al. 1999; Zapata et al. 2011) and typical shock velocities of $50-100 \mathrm{~km} \mathrm{~s}^{-1}$, a post-shock density of $10^{9} \mathrm{~cm}^{-3}$ (required for water maser action - Hollenbach et al. 2013) can be obtained for a pre-shock magnetic field of a $\sim 1-2 \mathrm{mG}^{11}$. According to the J-shock model of Hollenbach \& McKee (1979), the component perpendicular to the shock propagation (parallel to the shock front) is enhanced by a factor equal to the ratio between the post- and pre-shock densities (their Eq. (2.21)): $B_{\perp}=$ $B_{0 \perp} n_{\mathrm{s}} / n_{0}$. Therefore, after the shock passage, $B_{\perp}$ would be amplified to $100-200 \mathrm{mG}$ (assuming $n_{0}=10^{7} \mathrm{~cm}^{-3}, n_{\mathrm{s}}=10^{9} \mathrm{~cm}^{-3}$, and $\left.B_{0 \perp}=1-2 \mathrm{mG}\right)$. This is consistent with the range of values of the magnetic field strength measured with the $\mathrm{H}_{2} \mathrm{O}$ masers (Table 1).

Since according to the same model the component of the magnetic field parallel to the shock velocity does not vary (see Eq. (2.16) in Hollenbach \& McKee 1979), we can use this prediction to derive constraints on the strength of the parallel component in the preshock gas. The analysis in Sect. 4.2.1 indicates a maximum ratio $B_{\perp} / B_{\|} \approx 6$ in the post-shock region. Since $B_{\perp}$ is likely amplified by a factor of 100 in the fast shocks producing the water masers while $B_{\|}$remains unchanged, we can infer that $B_{0 \|} \geq 10 B_{0 \perp}$.

10 We note that the numerical factor is not scalar, but absorbs the unit of the ratio of densities with different exponents.

11 A model for C-type shocks provides magnetic fields of the same order of magnitude (see Eq. (4.5) of Kaufman \& Neufeld 1996). 
According to this crude analysis, we constrain the magnetic field strength in the pre-shock gas to $10-20 \mathrm{mG}$. This range is the right order of magnitude for the magnetic field derived from the synchrotron emission model by Reid et al. (1995), which infers a strength of the magnetic field $B_{0}=10 \mathrm{mG}^{12}$. Likewise, our estimate of magnetic field strength is also consistent with the value of $B_{\text {sky }}=17 \mathrm{mG}$ reported by Chen et al. (2012) from dust emission.

We stress that, even allowing a factor of 10 between $B_{0 \|}$ and $B_{0 \perp}, B_{\perp}$ would still dominate in the post-shock gas, consistent with an estimated range of 100-300 mG.

In summary, the magnetic field would evolve from having a dominant component parallel to the shock velocity in the preshock gas, with field strengths of the order of a few tens of $\mathrm{mG}$, to being mainly dominated by the perpendicular component of order of a few hundred of $\mathrm{mG}$ in the post-shock gas exciting the $\mathrm{H}_{2} \mathrm{O}$ masers ${ }^{13}$. The general implication of this finding is that in the undisturbed (i.e., not-shocked) circumstellar gas, the flow velocities would follow closely the magnetic field lines, while in the shocked gas the magnetic structure would be re-configured to be parallel to the shock front.

\section{Summary and conclusions}

We present the first VLBI polarization measurements of $\mathrm{H}_{2} \mathrm{O}$ masers in $\mathrm{W} 3\left(\mathrm{H}_{2} \mathrm{O}\right)$. This HMSF region contains the best known (archetypal) case of a synchrotron jet driven by an embedded high-mass YSO, the TW-object or TW-A, and therefore provides a good target for investigating the relation between the gas dynamics and the magnetic field in regulating massloss in HMSF. In particular, our polarimetric measurements of $\mathrm{H}_{2} \mathrm{O}$ masers have enabled us to infer the magnetic field strength and orientation, that can be directly compared to the kinematics of the molecular outflowing gas, on scales of order of tens to hundreds AU, among the smallest accessible scales in studies of HMSF.

The main findings of this work are the following:

1. We measured a linear polarization fraction varying in the range $0.9-42 \%$, making $\mathrm{W} 3\left(\mathrm{H}_{2} \mathrm{O}\right)$ the highest-polarized $\mathrm{H}_{2} \mathrm{O}$ maser source on VLBI scales known in the Galaxy. The masers show also circularly polarized emission, in the range $0.2-1.6 \%$.

2. On scales of order of one thousand AU or larger, the average magnetic field orientation probed by $\mathrm{H}_{2} \mathrm{O}$ masers is mainly E-W, well aligned with the axis of the synchrotron jet driven from the TW-A protostar, suggesting that the molecular masers may probe the magnetic field in the protostellar jet.

3. On smaller scales, 10 s to 100 s of $\mathrm{AU}$, a detailed comparison between the magnetic field orientations and the proper motions of the $\mathrm{H}_{2} \mathrm{O}$ masers observed at the center of the protostellar jet/outflow, reveals a misalignment between the magnetic field and the velocity vectors, which can be explained with an origin in magnetically supported shocks.

\footnotetext{
${ }^{12}$ We note that this value is constrained to no better than a factor of a few from the fit of the radio continuum data.

${ }_{13}$ The parallel component could dominate in the post-shock gas only if it were $>100 \mathrm{mG}$ in the pre-shock gas, that is, 2 orders of magnitude stronger than the pre-shock perpendicular component; a $100 \mathrm{mG}$ field in $10^{7} \mathrm{~cm}^{-3}$ gas is however implausibly high. Therefore, we can rule out that with $\mathrm{H}_{2} \mathrm{O}$ masers we are measuring the parallel component and we are dominated by the perpendicular component.
}

4. Since water masers emerge behind C- and/or J-type shocks, the shock passage alters the initial magnetic field configuration in the circumstellar gas, by compressing and enhancing the component of the magnetic field perpendicular to the shock velocity with respect to the parallel component (by a factor equal to the ratio between the post- and pre-shock densities: typically a 100). In the gas shocked by the synchrotron jet, we estimate a total field strength in the range $\sim 100-300 \mathrm{mG}$ (at densities of $10^{9} \mathrm{~cm}^{-3}$ ) and we conclude that fields of this order of magnitude are expected if the observed polarized water masers indeed emerge behind magnetically-supported shocks which compress the field component along the shock front.

5. Although the water maser polarization measurements alone cannot provide a direct measurement of the magnetic field properties in the quiescent (pre-shock) circumstellar gas, nevertheless by combining the information on the orientation and strength of the magnetic field (in the post-shock gas) with the knowledge of the maser velocities, we could constrain the magnetic field strength in the pre-shock circumstellar gas to $10-20 \mathrm{mG}$ (at densities of $10^{7} \mathrm{~cm}^{-3}$ ), which is consistent with previous estimates from a synchrotron jet model and dust polarization measurements.

6. In the pre-shock gas, we estimate a lower limit of 10 for the ratio of the parallel and perpendicular components of the magnetic field with respect to the proper motions. This indicates that the flow velocities follow closely the magnetic field lines and is suggestive of a local coupling between the magnetic field and the kinematics of the circumstellar gas in the $\mathrm{W} 3\left(\mathrm{H}_{2} \mathrm{O}\right)$ core and the protostellar jet from the TW-object.

This study demonstrates that, although $\mathrm{H}_{2} \mathrm{O}$ masers can naturally probe only the magnetic field in regions shocked by protostellar jets, by combining the knowledge of the 3D velocities and magnetic field orientation and strength, it is still possible to derive constraints on the properties of the magnetic field in the preshock circumstellar gas at the base of protostellar outflows. In particular, our results in $\mathrm{W} 3\left(\mathrm{H}_{2} \mathrm{O}\right)$ suggest the presence of a local coupling between the magnetic field and the gas kinematics, indicating that magnetic fields can be dynamical important in driving the gas outflowing from a high-mass protostar. Future studies based on VLBI multi-epoch observations of $\mathrm{H}_{2} \mathrm{O}$ masers in full polarization mode (which naturally provide magnetic field and velocity vectors in the same maser features), have the potential to open up a new window into the investigation of the role of magnetic fields in the gas dynamics of high-mass protostellar disk-jet systems, on scales of only tens to hundreds AU from the exciting massive protostar.

Acknowledgements. We are grateful to Dr F. Wyrowski and Dr K. Hachisuka for providing the $1.4 \mathrm{~mm}$ and the $8.4 \mathrm{GHz}$ continuum images, respectively. We are grateful to Dr K. Hachisuka for useful discussion on absolute proper motion measurements of water masers. C.G. acknowledges support from ERC synergy grant 610058 (Goddi et al. 2016). W.V. acknowledges support from ERC consolidator grant 614264 .

\section{References}

Alcolea, J., Menten, K. M., Moran, J. M., \& Reid, M. J. 1993, in Astrophysical Masers, eds. A. W. Clegg, \& G. E. Nedoluha, Lect. Not. Phys., 412 (Berlin: Springer Verlag), 225

Andre, P. 1996, in Radio Emission from the Stars and the Sun, eds. A. R. Taylor \& J. M. Paredes, ASP Conf. Ser., 93, 273

Anglada, G., Villuendas, E., Estalella, R., et al. 1998, AJ, 116, 2953 
Carrasco-González, C., Rodríguez, L. F., Anglada, G., et al. 2010, Science, 330, 1209

Chen, H.-R., Rao, R., Wilner, D. J., \& Liu, S.-Y. 2012, ApJ, 751, L13

Elitzur, M., Hollenbach, D. J., \& McKee, C. F. 1989, ApJ, 346, 983

Garay, G., Moran, J. M., \& Haschick, A. D. 1989, ApJ, 338, 244

Goddi, C., Falcke, H., Kramer, M., et al. 2016, ArXiv e-prints [arXiv: 1606.08879]

Goldreich, P., Keeley, D. A., \& Kwan, J. Y. 1973, ApJ, 179, 111

Greenhill, L. J., Goddi, C., Chandler, C. J., Matthews, L. D., \& Humphreys, E. M. L. 2013, ApJ, 770, L32

Hachisuka, K., Brunthaler, A., Menten, K. M., et al. 2006, ApJ, 645, 337

Hoare, M. G., Kurtz, S. E., Lizano, S., Keto, E., \& Hofner, P. 2007, Protostars and Planets V, 181

Hofner, P., Kurtz, S., Ellingsen, S. P., et al. 2011, ApJ, 739, L17

Hollenbach, D., \& McKee, C. F. 1979, ApJS, 41, 555

Hollenbach, D., Elitzur, M., \& McKee, C. F. 2013, ApJ, 773, 70

Imai, H., Horiuchi, S., Deguchi, S., \& Kameya, O. 2003, ApJ, 595, 285

Kaufman, M. J., \& Neufeld, D. A. 1996, ApJ, 456, 611

Marscher, A. P., Jorstad, S. G., Mattox, J. R., \& Wehrle, A. E. 2002, ApJ, 577, 85

Matthews, L. D., Greenhill, L. J., Goddi, C., et al. 2010, ApJ, 708, 80

Moscadelli, L., Goddi, C., Cesaroni, R., Beltrán, M. T., \& Furuya, R. S. 2007, A\&A, 472, 867

Moscadelli, L., Cesaroni, R., Sánchez-Monge, Á., et al. 2013, A\&A, 558, A145

Moscadelli, L., Sánchez-Monge, Á., Goddi, C., et al. 2016, A\&A, 585, A71
Nedoluha, G. E., \& Watson, W. D. 1992, ApJ, 384, 185

Reid, M. J., Argon, A. L., Masson, C. R., Menten, K. M., \& Moran, J. M. 1995, ApJ, 443, 238

Sarma, A. P., Troland, T. H., Crutcher, R. M., \& Roberts, D. A. 2002, ApJ, 580, 928

Sanna, A., Surcis, G., Moscadelli, L., et al. 2015, A\&A, 583, L3

Surcis, G., Vlemmings, W. H. T., Curiel, S., et al. 2011a, A\&A, 527, A48

Surcis, G., Vlemmings, W. H. T., Torres, R. M., van Langevelde, H. J., \& Hutawarakorn Kramer, B. 2011b, A\&A, 533, A47

Surcis, G., Vlemmings, W. H. T., van Langevelde, H. J., et al. 2014, A\&A, 565, L8

Torrelles, J. M., Patel, N. A., Gómez, J. F., et al. 2001, ApJ, 560, 853

Torrelles, J. M., Patel, N. A., Curiel, S., et al. 2011, MNRAS, 410, 627

Turner, J. L., \& Welch, W. J. 1984, ApJ, 287, L81

Vlemmings, W. H. T. 2006, A\&A, 445, 1031

Vlemmings, W. H. T. 2012, in Cosmic Masers - from OH to H0, eds. R. S Booth, W. H. T. Vlemmings, \& E. M. L. Humphreys, IAU Symp., 287, 31

Vlemmings, W. H. T., Diamond, P. J., van Langevelde, H. J., \& Torrelles, J. M. 2006, A\&A, 448, 597

Wardle, J. F. C., \& Kronberg, P. P. 1974, ApJ, 194, 249

Wilner, D. J., Reid, M. J., \& Menten, K. M. 1999, ApJ, 513, 775

Wyrowski, F., Schilke, P., Walmsley, C. M., \& Menten, K. M. 1999, ApJ, 514, L43

Zapata, L. A., Rodríguez-Garza, C., Rodríguez, L. F., Girart, J. M., \& Chen, H.-R. 2011, ApJ, 740, L19 


\section{Appendix A: Analysis of maser polarization data}

We analyzed the polarimetric data following three main steps: (i) identification of individual $\mathrm{H}_{2} \mathrm{O}$ maser features and measurement of their linear polarization fraction and polarization angle; (ii) determination of the orientation of the magnetic field on the plane of the sky; (iii) measurement of Zeeman splitting and magnetic field strength along the l.o.s.

The methodology is described in full detail in Surcis et al. (2011a,b). Here below we describe the main steps for completeness.

(i) To identify the water masers in the $\mathrm{W} 3\left(\mathrm{H}_{2} \mathrm{O}\right)$ region, first we searched for maser emission channel by channel, with a signal-to-noise ratio greater than 10-sigma noise level; then, we fitted the identified maser spots with a 2D Gaussian function (using the AIPS task IMFIT); and finally we defined individual maser features as collections of three or more maser spots in consecutive velocity channels within the full width half-maximum (FWHM) beamsize. Once identified all the maser features, the next step was to estimate the linear polarization fraction and the polarization angle in each of them. For this purpose, we produced spectra of total intensity $(I)$, polarized intensity (POLI), and polarization angle (POLA; see Sect. 2) at the position of each identified maser feature (integrating in a box $3 \times 3$ pixels). The linear polarization fraction $\left(P_{1}\right)$ and the linear polarization angle $(\chi)$ of a single feature are determined taking the average values in consecutive channels across its total intensity spectral profile with $P O L I \geq 5 \sigma$ (Fig. A. 1 shows a few examples). Table 1 reports the main parameters of all the maser features identified in the region.

(ii) The orientation or position angle of the magnetic field on the plane of the sky can be determined using the theory of polarized maser emission, as outlined in Goldreich et al. (1973). In particular, the relation between the measured polarization angle $\chi$ and the magnetic field angle on the sky depends on the angle between the maser propagation direction and the magnetic field orientation, $\theta$, with the linear polarization vector perpendicular to the field for $\theta>\theta_{\text {crit }}=55^{\circ}$, where $\theta_{\text {crit }}$ is the so-called Van Vleck angle, and parallel otherwise (Goldreich et al. 1973). $\theta$ can be determined from the emerging brightness temperature, $T_{\mathrm{b}} \Delta \Omega^{14}$, and the fractional linear polarization, $P_{1}$, of the $\mathrm{H}_{2} \mathrm{O}$ maser emission (Goldreich et al. 1973). $T_{\mathrm{b}} \Delta \Omega$ is estimated by fitting the spectral profiles of individual water maser emissions by using the FRTM code developed by Vlemmings (2006) and based on the model of Nedoluha \& Watson (1992). In this model, the spectral profiles of the total intensity, linear polarization, and circular polarization depend on $T_{\mathrm{b}} \Delta \Omega$, as well as the intrinsic thermal linewidth, $\Delta V_{\mathrm{i}}{ }^{15}$. The FRTM code uses a $\chi^{2}$-fitting analysis and provides in output $T_{\mathrm{b}} \Delta \Omega$ and $\Delta V_{\mathrm{i}}$ (refer to Surcis et al. 2011a for a detailed description). From $P_{1}$ and $T_{\mathrm{b}} \Delta \Omega$ we can then determine $\theta$ (the estimated values are reported in Col. 13 in Table D.1). We note that the estimated $\theta$ values have large negative (and small positive) error bars. This is a consequence of the probability calculation in the FRTM code. In particular, Vlemmings (2006) calculated the expected relation between $\theta$ and $P_{1}$ for different values of $T_{\mathrm{b}} \Delta \Omega$

\footnotetext{
${ }^{14}$ The emerging brightness temperature, $T_{\mathrm{b}} \Delta \Omega$, is defined as the product of the brightness temperature, $T_{\mathrm{b}}$, and the solid angle of the maser beam, $\Delta \Omega$.

${ }^{15} \Delta V_{\mathrm{i}}$ is basicaly the FWHM of the Maxwellian distribution of particle velocities.
}

and showed that there are typically three values of $\theta$ that produce the same linear polarization. For instance, looking at their Fig. 6, one could realize that a maser with $\theta \sim 50^{\circ}$ (for a non-saturated maser) would have the same $P_{1}$ as a maser with $\theta \sim 10^{\circ}$ or a maser with $\theta \sim 58^{\circ}$ (in which case the linear polarization angle on the sky flips). Therefore, for a given (low) saturation level and low polarization level, it is more likely to have a component with $\theta<55^{\circ}$, but the uncertainty interval toward the low end is very large.

(iii) The magnetic field strength along the l.o.s. can be calculated from the Zeeman-splitting measurements by using $B_{\text {l.o.s. }}=$ $\Delta V_{Z} \alpha_{Z}$, where $\alpha_{Z}$ is the Zeeman-splitting coefficient. This is done by fitting the total intensity and circular polarized spectra of the $\mathrm{H}_{2} \mathrm{O}$ masers identified in the high-spectral resolution $I$ and $V$ cubes (see Sect. 2 and Fig. A.2 for a few examples). The best estimates of $T_{\mathrm{b}} \Delta \Omega$ and $\Delta V_{\mathrm{i}}$ (as described in step ii) are included in the FRTM code to produce the $I$ and $V$ models used in the fitting procedure. The Zeeman splitting depends on $T_{\mathrm{b}} \Delta \Omega$, the FWHM of the maser total intensity profile, $\Delta v_{L}$, and the circular polarization fraction, $P_{\mathrm{V}}$. Once the field strength along the 1.o.s. is estimated, one can determine the total magnetic field strength from $B=B_{\text {l.o.s. }} / \cos \theta$.

A final element worth noting is that the degree of saturation affects the fractional linear polarization $P_{1}$. When the saturation sets in, the maser lines start to broaden, and the observed linewidth $\Delta v_{L}$ can become as large as $\Delta V_{\mathrm{i}}$. For a saturated maser, $T_{\mathrm{b}} \Delta \Omega$ and $\Delta V_{\mathrm{i}}$ cannot be properly disentangled by the model of Nedoluha \& Watson (1992), and the FRTM code only provides a lower limit for $T_{\mathrm{b}} \Delta \Omega$ and an upper limit for $\Delta V_{\mathrm{i}}$. As a result, the fitted values of $\theta$ are overestimated.

\section{Appendix B: Astrometry}

We did not observe in phase-referencing mode, therefore after self-calibration we cannot recover the information on the absolute position in our maser polarization maps. The positions of the identified $\mathrm{H}_{2} \mathrm{O}$ masers are relative to the strongest feature used for self-calibration: ID 018 at $-48.6 \mathrm{~km} \mathrm{~s}^{-1}$ and with peak flux density $\sim 2 \times 10^{3} \mathrm{Jy} \mathrm{beam}^{-1}$ (see Table D.1). In order to register our $\mathrm{H}_{2} \mathrm{O}$ maser polarization maps with previous $\mathrm{H}_{2} \mathrm{O}$ maser proper motion measurements (as well as with thermal emission maps), we used a two-steps process based on the astrometric measurements by Hachisuka et al. (2006). First, we aligned the brightest features $\left(>100 \mathrm{Jy}^{-1} \mathrm{bem}^{-1}\right)$ detected in both datasets towards cluster $d$ in the velocity range -48.4 to $-48.8 \mathrm{~km} \mathrm{~s}^{-1}$. These include our reference feature ID 018 and features ID 4, 5, 6, and 7 from Hachisuka et al. (2006, see their Table 2). The latter have a positional scatter of about 10 mas in RA and 40 mas in Dec, around the position: $\alpha_{2000}=2^{\mathrm{h}} 27^{\mathrm{m}} 04^{\mathrm{s}} .8362$ and $\delta_{2000}=$ $61^{\circ} 52^{\prime} 24^{\prime \prime} 607$, setting the accuracy of the registration between the two maser datasets at the level of several tens of mas. In order to refine such registration to higher accuracy, we shifted our polarized maps in such a fashion to minimize the distance among the persistent maser knots detected in the two datasets. For this purpose, we used only the maser cluster $a$ which displays more persistent, recognizable structures with respect to cluster $d$ (affected by strong shocks) over the five years separation between the two observations. We found that the positional offset that maximizes the overlap among the two maser distributions is: $\Delta x=-10$ mas, $\Delta y=+33$ mas (with respect to the 

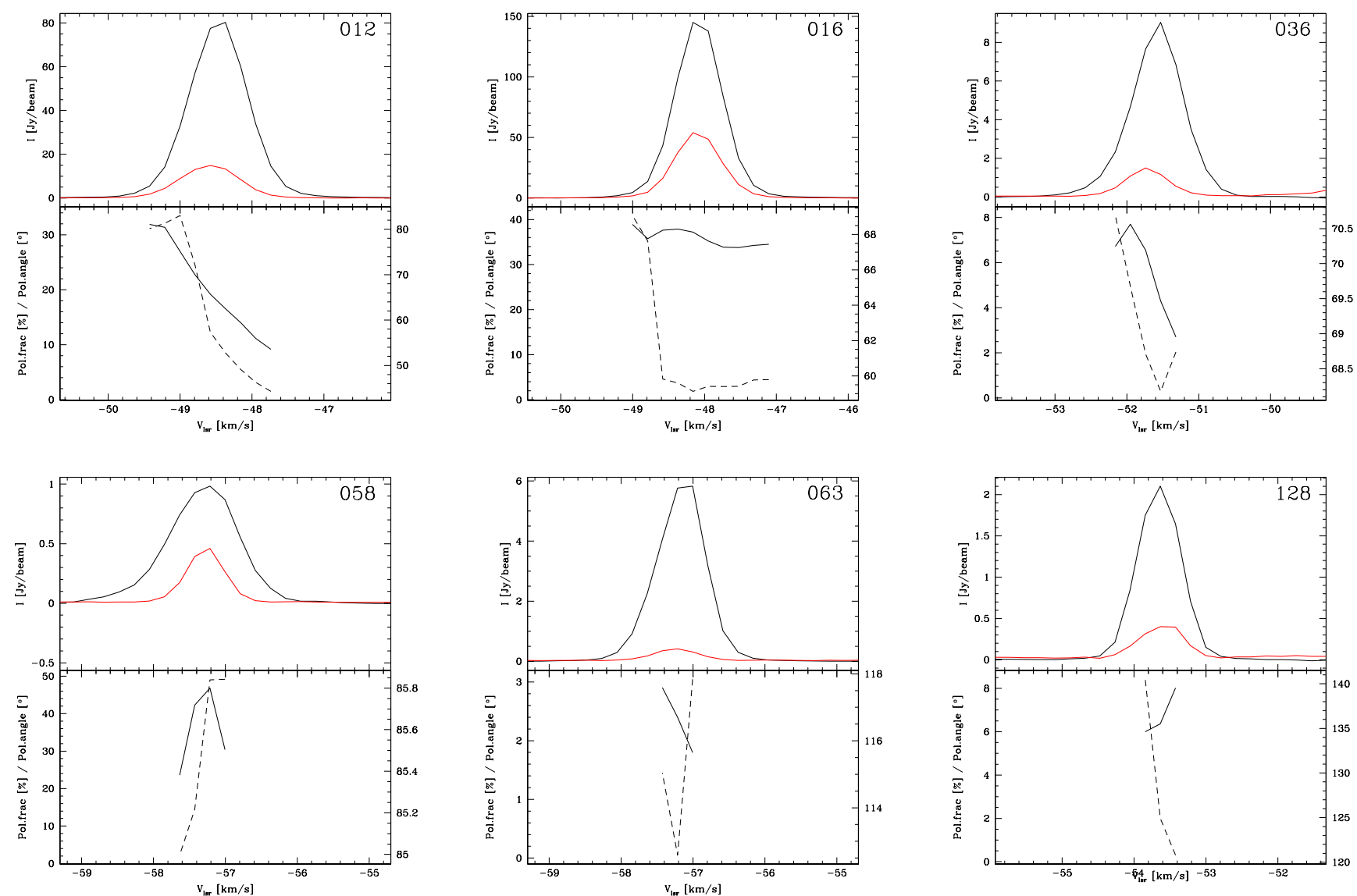

Fig. A.1. Total intensity ( $I$, black solid line) and linear polarization intensity (red solid line) spectra of the $\mathrm{H}_{2} \mathrm{O}$ maser features $012,016,036,058$, 063, and 128 (upper panel). The linear polarization intensity spectra have been multiplied by a factor of three for 036, 063, and 128. The linear polarization fraction (black solid line, left scale) and the linear polarization angle (dashed black line, right scale) are also shown (lower panel).
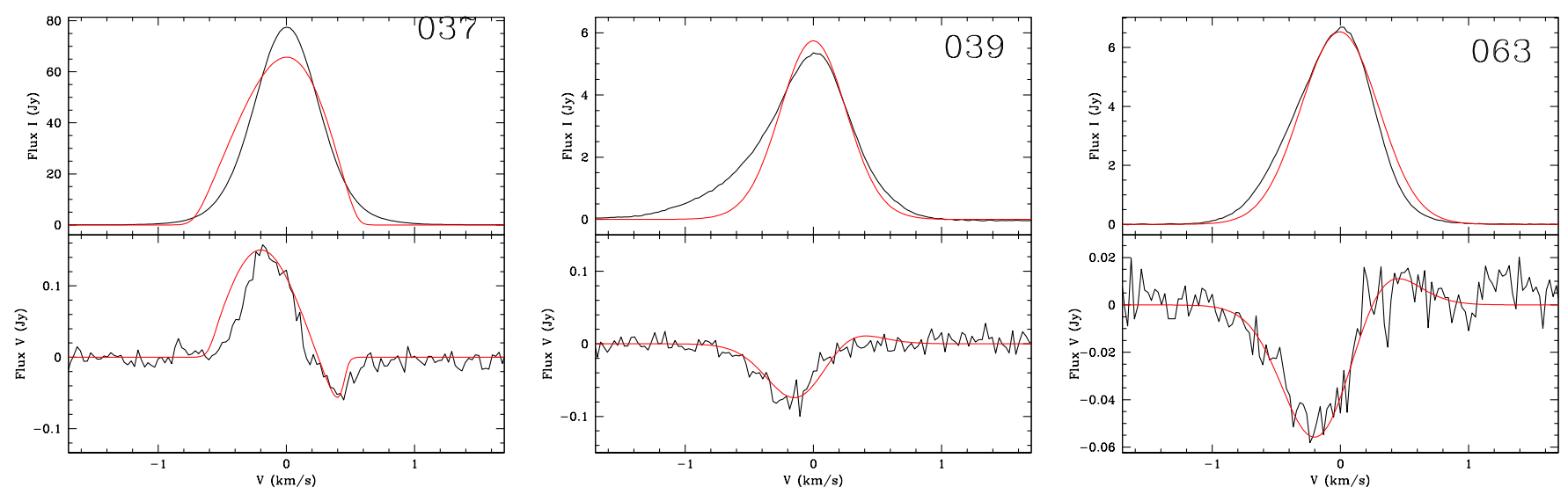

Fig. A.2. Total intensity (I, upper panel) and circular polarization intensity ( $V$, lower panel) spectra for the $\mathrm{H}_{2} \mathrm{O}$ maser features 037 , 039,063 . The thick red line shows the best-fit models of $I$ and $V$ emission obtained using the FRTM code (see Appendix A). The maser features are centered on zero velocity.

position: $\alpha_{2000}=2^{\mathrm{h}} 27^{\mathrm{m}} 04^{\mathrm{s}} .8362$ and $\left.\delta_{2000}=61^{\circ} 52^{\prime} 24^{\prime \prime} 607\right)$. Incidentally, we notice that applying this offset brings in coincidence (within 1 mas) our strongest feature at $-48.6 \mathrm{~km} \mathrm{~s}^{-1}$ (ID 018 in Table D.1) with the strongest feature detected by Hachisuka et al. (2006, ID 6 in their Table D.1).

According to this analysis, we conclude that our absolute astrometry has an accuracy of the order of a few milliarcseconds.

\section{Appendix C: Geometry of the magnetic field with respect to the proper motions of water masers}

According to the definition of $\theta$ as the angle between the magnetic field vector $\boldsymbol{B}$ and the line of sight (see Sect. 3.1), we can 
C. Goddi et al.: The magnetic field in a protostellar synchrotron jet

define:

$B_{\text {l.o.s. }}=B \cos (\theta), \quad B_{\text {sky }}=B \sin (\theta)$,

where $B_{\text {l.o.s. }}$ and $B_{\text {sky }}$ are the components of the magnetic field $\boldsymbol{B}$ along the line-of-sight and projected in the plane of the sky, respectively. If now indicate with $\phi$ the angle between $B_{\text {sky }}$ and the proper motion orientation, we can decompose $B_{\text {sky }}$ in the two components:

$B_{\mathrm{sky}_{\|}}=B \sin (\theta) \cos (\phi), \quad B_{\mathrm{sky}_{\perp}}=B \sin (\theta) \sin (\phi)$.

Merging the expressions above, we can write the components of $\boldsymbol{B}$ parallel, $B_{\|}$, and perpendicular, $B_{\perp}$, to the proper motion orientation as follows:

$B_{\|} \equiv B_{\text {sky } \|}=B \sin (\theta) \cos (\phi)$,

$B_{\perp}=\sqrt{\left(B_{\text {l.o.s. }}\right)^{2}+\left(B_{\text {sky }}\right)^{2}}=\sqrt{(B \cos (\theta))^{2}+(B \sin (\theta) \sin (\phi))^{2}}$.
Consequently, we can derive a ratio between these components as:

$B_{\perp} / B_{\|}=\sqrt{\cot ^{2}(\theta) / \cos ^{2}(\phi)+\tan ^{2}(\phi)}$. 


\section{Appendix D: Additional table}

Table D.1. Parameters of the 22- $\mathrm{GHz} \mathrm{H}_{2} \mathrm{O}$ maser features detected in $\mathrm{W} 3(\mathrm{OH})$.

\begin{tabular}{|c|c|c|c|c|c|c|c|c|c|c|c|c|}
\hline $\begin{array}{l}\text { (1) } \\
\text { ID }\end{array}$ & $\begin{array}{c}(2) \\
\text { RA }^{a} \\
\text { offset } \\
(\operatorname{arcsec})\end{array}$ & $\begin{array}{c}(3) \\
\text { Dec }^{a} \\
\text { offset } \\
(\operatorname{arcsec})\end{array}$ & $\begin{array}{c}(4) \\
\text { Peak flux } \\
\text { density }(I) \\
\text { (Jy/beam) }\end{array}$ & $\begin{array}{c}(5) \\
V_{\mathrm{lsr}} \\
\left(\mathrm{km} \mathrm{s}^{-1}\right)\end{array}$ & $\begin{array}{c}(6) \\
\Delta v_{\mathrm{L}} \\
\left(\mathrm{km} \mathrm{s}^{-1}\right)\end{array}$ & $\begin{array}{l}(7) \\
P_{1} \\
(\%)\end{array}$ & $\begin{array}{l}(8) \\
\chi\end{array}$ & $\begin{array}{c}(9) \\
\Delta V_{\mathrm{i}}^{b} \\
\left(\mathrm{~km} \mathrm{~s}^{-1}\right)\end{array}$ & $\begin{array}{c}(10) \\
T_{\mathrm{b}} \Delta \Omega^{b} \\
(\log \mathrm{K} s \mathrm{~s})\end{array}$ & $\begin{array}{c}(11) \\
P_{\mathrm{V}} \\
(\%)\end{array}$ & $\begin{array}{l}(12) \\
B_{\text {l.o.s. }} \\
(\mathrm{mG})\end{array}$ & $\begin{array}{c}(13) \\
\theta^{c}\end{array}$ \\
\hline \multicolumn{13}{|c|}{ Cluster " $d$ " } \\
\hline 001 & 0.0897 & -0.2034 & 0.1 & -46.26 & 0.91 & - & - & - & - & - & - & - \\
\hline 002 & 0.0584 & -0.2000 & 0.06 & -46.47 & 0.92 & - & - & - & - & - & - & - \\
\hline 003 & 0.0523 & -0.0694 & 0.8 & -45.63 & 1.29 & - & - & - & - & - & - & - \\
\hline 004 & 0.0410 & -0.1185 & 2.9 & -46.47 & 0.86 & - & - & - & - & - & - & - \\
\hline 005 & 0.0397 & -0.0348 & 200.2 & -49.84 & 0.69 & $17.7 \pm 3.5$ & $79 \pm 3$ & $1.0_{-1.4}^{+0.5}$ & $10.8_{-2.4}^{+0.4}$ & - & - & $90_{-9}^{+9}$ \\
\hline 006 & 0.0393 & -0.0338 & 1.7 & -50.48 & 0.87 & - & - & - & - & - & - & - \\
\hline 007 & 0.0378 & -0.0967 & 1.5 & -47.32 & 0.87 & - & - & - & - & - & - & - \\
\hline 008 & 0.0276 & -0.0182 & 7.2 & -49.52 & 0.93 & $42 \pm 19$ & $-60 \pm 7$ & $2.2_{-0.6}^{+1.2}$ & $6.0_{-0.7}^{+2.1}$ & - & - & $90_{-65}^{+65}$ \\
\hline 009 & 0.0256 & -0.0462 & 27.3 & -48.16 & 0.89 & - & - & - & - & - & - & - \\
\hline 010 & 0.0247 & -0.0453 & 6.4 & -48.58 & 1.03 & $19.3 \pm 6.5$ & $-72 \pm 5$ & $1.3_{-1.2}^{+0.6}$ & $11.1_{-0.1}^{+3.2}$ & - & - & $90_{-45}^{+45}$ \\
\hline 011 & 0.0214 & -0.1055 & 204.7 & -48.79 & 0.98 & $10.2 \pm 1.2$ & $+63 \pm 7$ & $1.9_{-0.5}^{+0.7}$ & $10.6_{-0.3}^{+0.3}$ & - & - & $84_{-8}^{+3}$ \\
\hline 012 & 0.0205 & -0.1066 & 80.4 & -48.37 & 0.91 & $19.2 \pm 6.5$ & $+57 \pm 16$ & $1.1_{-1.3}^{-0.5}$ & $11.1_{-2.9}^{+0.3}$ & 0.2 & $-70 \pm 13$ & $90_{-38}^{+38}$ \\
\hline 013 & 0.0198 & -0.1077 & 23.5 & -46.47 & 0.83 & $3.6 \pm 0.7$ & $+82 \pm 2$ & $3.5_{-1.1}^{+05}$ & $9.7_{-1.0}^{+1.1}$ & - & - & $74_{-32}^{+16}$ \\
\hline 014 & 0.0129 & -0.0292 & 3.1 & -47.74 & 0.85 & - & - & - & - & - & - & - \\
\hline 015 & 0.0078 & -0.0455 & 5.9 & -47.95 & 0.67 & - & - & - & - & - & - & - \\
\hline 016 & 0.0022 & -0.0199 & 145.1 & -48.16 & 0.75 & $35.8 \pm 1.7$ & $+60 \pm 1$ & $1.0_{-0.2}^{+1.2}$ & $11.0_{-1.0}^{+0.2}$ & - & - & $90_{-3}^{+3}$ \\
\hline 017 & 0.0021 & -0.0199 & 0.2 & -50.90 & 3.11 & - & - & - & - & - & - & - \\
\hline 018 & 0.0000 & 0.0000 & 2050.3 & -48.58 & 0.95 & $15.0 \pm 2.7$ & $+6 \pm 10$ & $1.0_{-0.5}^{+1.5}$ & $11.2_{-1.6}^{+0.2}$ & 0.2 & $+59 \pm 9$ & $90_{-8}^{+8}$ \\
\hline 019 & -0.0034 & 0.0013 & 60.1 & -49.21 & 0.72 & $25.4 \pm 3.0$ & $-35 \pm 2$ & $1.1_{-0.3}^{+1.6}$ & $10.9_{-2.4}^{+0.4}$ & 0.4 & $-120 \pm 20$ & $90_{-6}^{+6}$ \\
\hline 020 & -0.0355 & 0.0627 & 10.0 & -49.42 & 0.54 & $36.1 \pm 3.3$ & $-37 \pm 1$ & $\begin{array}{l}1.0_{-0.8}^{+0.8} \\
\text { +0.8 }\end{array}$ & $11.0_{-2.7}^{+0.4}$ & - & - & $90_{-5}^{+5}$ \\
\hline 021 & -0.1391 & -0.1918 & 34.2 & -50.48 & 0.58 & $15.4 \pm 1.0$ & $-11 \pm 69$ & $\begin{array}{l}1.0_{-0.8}^{+0.8} \\
\text { +0.8 }\end{array}$ & $10.8_{-0.5}^{+0.3}$ & - & - & $90_{-5}^{+5}$ \\
\hline 022 & -0.2376 & -0.1840 & 6.8 & -47.95 & 1.43 & - & - & - & - & - & - & - \\
\hline 023 & -0.2407 & -0.1861 & 0.3 & -47.11 & 0.81 & - & - & - & - & - & - & - \\
\hline 024 & -0.2489 & -0.2023 & 1.0 & -59.95 & 0.73 & - & - & - & - & - & - & - \\
\hline 025 & -0.2590 & -0.2059 & 1.6 & -60.16 & 0.81 & - & - & - & - & - & - & - \\
\hline 026 & -0.2598 & -0.2051 & 0.2 & -59.11 & 0.61 & - & - & - & - & - & - & - \\
\hline 027 & -0.2615 & -0.2054 & 0.3 & -59.74 & 0.76 & - & - & - & - & - & - & - \\
\hline 028 & -0.2625 & -0.2049 & 0.08 & -58.90 & 0.70 & - & - & - & - & - & - & - \\
\hline 029 & -0.6072 & 0.0486 & 1.8 & -43.95 & 0.53 & - & - & - & - & - & - & - \\
\hline 030 & -0.6344 & 0.0427 & 0.1 & -54.06 & 1.13 & - & - & - & - & - & - & - \\
\hline 031 & -0.6354 & 0.0438 & 5.8 & -58.90 & 0.89 & - & - & - & - & - & - & - \\
\hline 032 & -0.6358 & 0.0461 & 9.2 & -56.16 & 0.71 & $1.7 \pm 0.2$ & $+62 \pm 3$ & $3.7_{-0.8}^{+0.4}$ & $9.3_{-1.6}^{+1.2}$ & - & - & $62_{-47}^{+5}$ \\
\hline 033 & -0.6366 & 0.0439 & 3.4 & -58.90 & 0.82 & - & - & - & - & - & - & - \\
\hline 034 & -0.6371 & 0.0439 & 7.0 & -59.32 & 0.79 & - & - & - & - & - & - & - \\
\hline 035 & -0.6380 & 0.0438 & 1.9 & -59.32 & 0.73 & - & - & - & - & - & - & - \\
\hline 036 & -0.6416 & 0.0537 & 9.0 & -51.53 & 0.76 & $6.6 \pm 1.2$ & $+69 \pm 1$ & $3.5_{-0.9}^{+0.5}$ & $8.7_{-0.3}^{+3.1}$ & 0.8 & $-356 \pm 107$ & $90_{-18}^{+18}$ \\
\hline 037 & -0.6425 & 0.0542 & 71.8 & -51.74 & 0.61 & $1.4 \pm 0.1$ & $+69 \pm 1$ & $1.0_{-0.1}^{+0.8}$ & $10.7_{-0.6}^{+0.2}$ & 0.3 & $-60 \pm 11$ & $\mathbf{5 1}_{-37}^{+1}$ \\
\hline 038 & -0.6428 & 0.0476 & 1.6 & -60.58 & 0.87 & - & - & - & - & - & - & - \\
\hline 039 & -0.6433 & 0.0546 & 4.8 & -51.53 & 0.68 & $11.8 \pm 3.0$ & $+88 \pm 1$ & $2.5_{-1.0}^{+0.7}$ & $8.1_{-2.0}^{+0.5}$ & 1.6 & $+411 \pm 114$ & $90_{-14}^{+14}$ \\
\hline 040 & -0.6437 & 0.0498 & 0.7 & -55.32 & 0.77 & - & - & - & - & - & - & - \\
\hline 041 & -0.6453 & 0.0501 & 0.7 & -55.74 & 0.70 & - & - & - & - & - & - & - \\
\hline 042 & -0.6515 & 0.0535 & 0.2 & -54.27 & 0.84 & - & - & - & - & - & - & - \\
\hline
\end{tabular}

Notes. The number ID of the detected maser features is reported in Col. 1. The positions, Cols. 2 and 3 , refer to the brightest $\mathrm{H}_{2} \mathrm{O}$ maser feature 018 that was used to self-calibrate the data. The peak flux density $(I)$, the LSR velocity $\left(V_{\mathrm{lsr}}\right)$, and the FWHM $\left(\Delta v_{\mathrm{L}}\right)$ of the total intensity spectra of the maser features are reported in Cols. 4, 5, and 6, respectively; $I, V_{\mathrm{LSR}}$, and $\Delta v_{\mathrm{L}}$ are obtained using a Gaussian fit. The mean linear polarization fraction, $P_{1}$, and the mean linear polarization angles, $\chi$, are instead reported in Cols. 7 and 8 , respectively. We determined $P_{1}$ and $\chi$ of each $\mathrm{H}_{2} \mathrm{O}$ maser feature by considering (more than two) consecutive channels across the total intensity spectrum for which the polarized intensity is $\geq 5 \sigma$. In Col. 9 we report the intrinsic thermal linewidth of the maser $\Delta V_{\mathrm{i}}$. In Col. 10 we report the emerging brightness temperature $T_{\mathrm{b}} \Delta \Omega$, which is the product of the brightness temperature $T_{\mathrm{b}}$ and the solid angle of the maser beam $\Delta \Omega{ }^{(a)}$ The reference position is assumed to be $\alpha_{2000}=2^{\mathrm{h}} 27^{\mathrm{m}} 04^{\mathrm{s}} .8362$ and $\delta_{2000}=61^{\circ} 52^{\prime} 24^{\prime \prime} 607$ (an additional offset of $\Delta x=-10$ mas, $\Delta y=+33$ mas is also assumed in the absolute astrometry; see Appendix B). ${ }^{(b)}$ The best-fitting results obtained by using a model based on the radiative transfer theory of water masers (Vlemmings 2006; Surcis et al. 2011a). The errors were determined by analyzing the full probability distribution function. ${ }^{(c)}$ The angle $\theta$ between the magnetic field and the maser propagation direction is determined by using the observed $P_{1}$ and the fitted $T_{\mathrm{b}} \Delta \Omega$. The errors were determined by analyzing the full probability distribution function. We use boldface for values for which the magnetic field is more likely parallel to $\chi$. 
Table D.1. continued.

\begin{tabular}{|c|c|c|c|c|c|c|c|c|c|c|c|c|}
\hline $\begin{array}{l}\text { (1) } \\
\text { ID }\end{array}$ & $\begin{array}{c}(2) \\
\text { RA }^{a} \\
\text { offset } \\
(\operatorname{arcsec})\end{array}$ & $\begin{array}{c}(3) \\
\text { Dec }^{a} \\
\text { offset } \\
(\operatorname{arcsec})\end{array}$ & $\begin{array}{c}(4) \\
\text { Peak flux } \\
\text { density }(I) \\
(\text { Jy/beam })\end{array}$ & $\begin{array}{c}(5) \\
V_{\mathrm{lsr}} \\
\left(\mathrm{km} \mathrm{s}^{-1}\right)\end{array}$ & $\begin{array}{c}(6) \\
\Delta v_{\mathrm{L}} \\
\left(\mathrm{km} \mathrm{s}^{-1}\right)\end{array}$ & $\begin{array}{l}(7) \\
P_{1} \\
(\%)\end{array}$ & $\begin{array}{l}(8) \\
\chi \\
\left({ }^{\circ}\right)\end{array}$ & $\begin{array}{c}(9) \\
\Delta V_{\mathrm{i}}^{b} \\
\left(\mathrm{~km} \mathrm{~s}^{-1}\right)\end{array}$ & $\begin{array}{c}(10) \\
T_{\mathrm{b}} \Delta \Omega^{b} \\
(\log \mathrm{K} \mathrm{sr})\end{array}$ & $\begin{array}{l}(11) \\
P_{\mathrm{V}} \\
(\%)\end{array}$ & $\begin{array}{l}(12) \\
B_{\text {l.o.s. }} \\
(\mathrm{mG})\end{array}$ & $\begin{array}{c}(13) \\
\theta^{c} \\
\left({ }^{\circ}\right)\end{array}$ \\
\hline \multicolumn{13}{|c|}{ Cluster " $a$ " } \\
\hline 043 & -0.6520 & 0.0590 & 28.0 & -51.53 & 0.64 & $0.9 \pm 0.1$ & $+69 \pm 4$ & $1.6_{-07}^{+0.5}$ & $10.1_{-10}^{+0.7}$ & - & - & $60_{-46}^{+2}$ \\
\hline 044 & -0.6622 & 0.0630 & 2.3 & -52.16 & 0.53 & - & - & -0.7 & - & - & - & -46 \\
\hline 045 & -0.6633 & 0.0633 & 2.4 & -52.58 & 0.53 & - & - & - & - & - & - & - \\
\hline 046 & -0.6643 & 0.0624 & 0.9 & -53.42 & 0.56 & - & - & - & - & - & - & - \\
\hline 047 & -0.6650 & 0.0621 & 2.2 & -53.21 & 1.06 & - & - & - & - & - & - & - \\
\hline 048 & -0.6650 & 0.0636 & 3.9 & -53.00 & 0.50 & - & - & - & - & - & - & - \\
\hline 049 & -0.666 & 0.0624 & 5.3 & -53.63 & 0.56 & - & - & - & - & - & - & - \\
\hline 050 & -0.6665 & 0.0647 & 11.5 & -53.00 & 0.56 & $1.2 \pm 0.1$ & $+65 \pm 1$ & $1.2_{-0.1}^{+0.3}$ & $10.4_{-0.3}^{+0.2}$ & - & - & $51_{-35}^{+1}$ \\
\hline 051 & -0.6667 & 0.0648 & 11.0 & -53.00 & 0.54 & $1.3 \pm 0.1$ & $+63 \pm 1$ & $1.2_{-0.2}^{-0.1}$ & $10.3_{-0.3}^{+0.3}$ & 0.4 & $-84 \pm 35$ & $49_{-32}^{+10}$ \\
\hline 052 & -0.6676 & 0.0649 & 4.1 & -53.00 & 0.67 & - & - & $-^{-0.2}$ & $--^{-0.3}$ & - & - & - \\
\hline 053 & -0.7339 & -0.1245 & 0.5 & -50.69 & 0.88 & - & - & - & - & - & - & - \\
\hline 054 & -0.7948 & -0.1137 & 0.3 & -58.69 & 0.70 & - & - & - & - & - & - & - \\
\hline 055 & -0.7976 & -0.1132 & 0.6 & -58.48 & 0.74 & - & - & - & - & - & - & - \\
\hline 056 & -0.8079 & -0.0662 & 12.0 & -60.58 & 0.84 & - & - & - & - & - & - & - \\
\hline 057 & -0.8112 & -0.0680 & 60.9 & -57.22 & 0.61 & $6.0 \pm 0.5$ & $-81 \pm 46$ & $1.4_{-0.9}^{+0.6}$ & $10.3_{-19}^{+0.9}$ & - & - & $79_{-7}^{+7}$ \\
\hline 058 & -0.8128 & -0.0679 & 1.0 & -57.21 & 0.98 & $42.3 \pm 8.2$ & $+86 \pm 1$ & $1.7_{-0.5}^{-1.9}$ & $8.3_{-2.0}^{+0.9}$ & - & - & $90_{-47}^{+47}$ \\
\hline 059 & -0.8129 & -0.0678 & 1.0 & -57.22 & 0.98 & - & - & - & - & - & - & - \\
\hline 060 & -0.8169 & -0.0628 & 1.8 & -48.37 & 1.20 & $22.3 \pm 4.0$ & $+63 \pm 3$ & $1.5_{-0.3}^{+1.5}$ & $8.4_{-0.1}^{+2.6}$ & - & - & $90_{-8}^{+8}$ \\
\hline 061 & -0.8170 & -0.0629 & 1.3 & -49.63 & 2.12 & - & - & $-^{-0.3}$ & - & - & - & - \\
\hline 062 & -0.8172 & -0.0692 & 1.3 & -57.00 & 0.57 & - & - & - & - & - & - & - \\
\hline 063 & -0.8182 & -0.0701 & 5.8 & -57.00 & 0.61 & $2.4 \pm 0.6$ & $-65 \pm 3$ & $3.0_{-0.7}^{+0.6}$ & $8.8_{-1.9}^{+0.7}$ & 0.9 & $+360 \pm 120$ & $90_{-48}^{+48}$ \\
\hline 064 & -0.8199 & -0.0643 & 1.7 & -56.58 & 1.33 & - & - & - & - & - & - & -48 \\
\hline 065 & -0.8206 & -0.0643 & 1.0 & -49.84 & 3.31 & - & - & - & - & - & - & - \\
\hline 066 & -0.8213 & -0.0644 & 1.1 & -47.32 & 1.86 & - & - & - & - & - & - & - \\
\hline 067 & -0.8227 & -0.0011 & 1.7 & -56.37 & 0.63 & - & - & - & - & - & - & - \\
\hline 068 & -0.8247 & -0.0706 & 0.4 & -56.79 & 0.56 & - & - & - & - & - & - & - \\
\hline 069 & -0.8259 & -0.0003 & 4.0 & -50.27 & 1.05 & $5.0 \pm 0.1$ & $-66 \pm 2$ & $1.3_{-1.4}^{+0.4}$ & $11.0_{-1.1}^{+0.6}$ & - & - & $67_{-2}^{+15}$ \\
\hline 070 & -0.8261 & -0.0706 & 0.8 & -55.95 & 1.24 & - & - & - & $-^{-1.1}$ & - & - & - \\
\hline 071 & -0.8275 & -0.0689 & 1.8 & -52.37 & 0.71 & - & - & - & - & - & - & - \\
\hline 072 & -0.8278 & -0.0714 & 0.8 & -55.95 & 0.74 & - & - & - & - & - & - & - \\
\hline 073 & -0.8284 & -0.0713 & 1.5 & -55.95 & 0.75 & - & - & - & - & - & - & - \\
\hline 074 & -0.8323 & -0.0709 & 0.08 & -54.69 & 0.73 & - & - & - & - & - & - & - \\
\hline 075 & -0.8370 & -0.0709 & 0.2 & -54.90 & 1.14 & - & - & - & - & - & - & - \\
\hline 076 & -0.8405 & -0.0019 & 0.8 & -43.53 & 0.68 & - & - & - & - & - & - & - \\
\hline 077 & -0.8435 & -0.0021 & 1.2 & -56.58 & 0.67 & - & - & - & - & - & - & - \\
\hline 078 & -0.8440 & -0.0015 & 0.6 & -55.53 & 1.10 & - & - & - & - & - & - & - \\
\hline 079 & -0.8443 & -0.0025 & 3.6 & -56.58 & 0.93 & - & - & - & - & - & - & - \\
\hline 080 & -0.8450 & -0.0040 & 0.9 & -58.69 & 0.70 & - & - & - & - & - & - & - \\
\hline 081 & -0.8458 & -0.0756 & 0.7 & -55.32 & 0.74 & - & - & - & - & - & - & - \\
\hline 082 & -0.8474 & -0.0754 & 0.09 & -59.11 & 0.83 & - & - & - & - & - & - & - \\
\hline 083 & -0.8499 & -0.0748 & 0.2 & -59.74 & 0.65 & - & - & - & - & - & - & - \\
\hline 084 & -0.8684 & -0.0666 & 1.6 & -60.58 & 1.16 & - & - & - & - & - & - & - \\
\hline 085 & -0.9286 & -0.0048 & 0.07 & -55.74 & 0.68 & - & - & - & - & - & - & - \\
\hline 086 & -0.9455 & -0.0023 & 1.6 & -56.79 & 0.70 & - & - & - & - & - & - & - \\
\hline 087 & -0.9481 & -0.0027 & 0.5 & -57.85 & 1.40 & - & - & - & - & - & - & - \\
\hline 088 & -0.9491 & 0.0122 & 0.3 & -55.11 & 0.70 & - & - & - & - & - & - & - \\
\hline 089 & -0.9501 & 0.0130 & 3.1 & -53.85 & 0.63 & - & - & - & - & - & - & - \\
\hline 090 & -0.9513 & 0.0132 & 0.5 & -54.48 & 0.66 & - & - & - & - & - & - & - \\
\hline 091 & -0.9540 & 0.0152 & 6.5 & -50.48 & 0.69 & - & - & - & - & - & - & - \\
\hline 092 & -0.9560 & 0.0151 & 15.7 & -53.85 & 1.90 & $1.4 \pm 0.1$ & $+69 \pm 2$ & $2.1_{-1.9}^{+0.1}$ & $10.8_{-1.8}^{+0.2}$ & - & - & $59_{-46}^{+1}$ \\
\hline 093 & -0.9563 & 0.0154 & 7.6 & -52.37 & 2.00 & - & - & ${ }^{-1.9}$ & $-^{-1.8}$ & - & - & - \\
\hline 094 & -0.9565 & -0.0485 & 0.2 & -55.53 & 0.81 & - & - & - & - & - & - & - \\
\hline 095 & -0.9568 & -0.0484 & 1.1 & -57.85 & 0.95 & - & - & - & - & - & - & - \\
\hline 096 & -0.9571 & -0.0479 & 0.9 & -57.85 & 0.63 & - & - & - & - & - & - & - \\
\hline 097 & -0.9574 & -0.0480 & 0.04 & -55.74 & 0.52 & - & - & - & - & - & - & - \\
\hline 098 & -0.9578 & 0.0139 & 2.4 & -43.10 & 0.80 & - & - & - & - & - & - & - \\
\hline
\end{tabular}


Table D.1. continued.

\begin{tabular}{|c|c|c|c|c|c|c|c|c|c|c|c|c|}
\hline $\begin{array}{l}\text { (1) } \\
\text { ID }\end{array}$ & $\begin{array}{c}(2) \\
\text { RA }^{a} \\
\text { offset } \\
(\operatorname{arcsec})\end{array}$ & $\begin{array}{c}(3) \\
\operatorname{Dec}^{a} \\
\text { offset } \\
(\operatorname{arcsec})\end{array}$ & $\begin{array}{c}(4) \\
\text { Peak flux } \\
\text { density }(I) \\
\text { (Jy/beam) }\end{array}$ & $\begin{array}{c}(5) \\
V_{\mathrm{lsr}} \\
\left(\mathrm{km} \mathrm{s}^{-1}\right)\end{array}$ & $\begin{array}{c}(6) \\
\Delta v_{\mathrm{L}} \\
\left(\mathrm{km} \mathrm{s}^{-1}\right)\end{array}$ & $\begin{array}{l}(7) \\
P_{1} \\
(\%)\end{array}$ & $\begin{array}{l}(8) \\
\chi \\
\left({ }^{\circ}\right)\end{array}$ & $\begin{array}{c}(9) \\
\Delta V_{\mathrm{i}}^{b} \\
\left(\mathrm{~km} \mathrm{~s}^{-1}\right)\end{array}$ & $\begin{array}{c}(10) \\
T_{\mathrm{b}} \Delta \Omega^{b} \\
(\log \mathrm{K} \mathrm{sr})\end{array}$ & $\begin{array}{l}(11) \\
P_{\mathrm{V}} \\
(\%)\end{array}$ & $\begin{array}{l}(12) \\
B_{\text {l.o.s. }} \\
(\mathrm{mG})\end{array}$ & $\begin{array}{l}(13) \\
\theta^{c} \\
\left({ }^{\circ}\right)\end{array}$ \\
\hline 099 & -0.9582 & 0.0140 & 2.5 & -43.32 & 0.92 & - & - & - & - & - & - & - \\
\hline 100 & -0.9591 & 0.0141 & 3.1 & -43.74 & 0.90 & - & - & - & - & - & - & - \\
\hline 101 & -0.9595 & 0.0142 & 4.7 & -44.79 & 1.08 & - & - & - & - & - & - & - \\
\hline 102 & -0.9596 & 0.0135 & 1.2 & -58.06 & 1.02 & - & - & - & - & - & - & - \\
\hline 103 & -0.9603 & 0.0162 & 0.6 & -51.32 & 0.58 & - & - & - & - & - & - & - \\
\hline 104 & -0.9606 & 0.0145 & 24.2 & -45.21 & 0.76 & $0.9 \pm 0.1$ & $+69 \pm 1$ & $1.3_{-0.3}^{+1.5}$ & $10.6_{-0.3}^{+0.1}$ & 0.6 & $+169 \pm 34$ & $55_{-41}^{+3}$ \\
\hline 105 & -0.9611 & 0.0156 & 17.2 & -53.85 & 0.81 & $1.5 \pm 0.1$ & $+86 \pm 7$ & $1.3_{-0.2}^{+0.3}$ & $10.7_{-0.9}^{-0.4}$ & 0.5 & $+161 \pm 41$ & $60_{-45}^{+11}$ \\
\hline 106 & -0.9615 & 0.0149 & 4.5 & -45.63 & 0.78 & - & - & - & - & - & - & - \\
\hline 107 & -0.9620 & 0.0167 & 5.5 & -51.74 & 0.77 & - & - & - & - & - & - & - \\
\hline 108 & -0.9627 & 0.0157 & 0.2 & -46.90 & 0.65 & - & - & - & - & - & - & - \\
\hline 109 & -0.9628 & 0.0158 & 7.7 & -49.84 & 0.82 & - & - & - & - & - & - & - \\
\hline 110 & -0.9632 & 0.0139 & 0.3 & -42.89 & 0.95 & - & - & - & - & - & - & - \\
\hline 111 & -0.9632 & 0.0168 & 2.6 & -51.32 & 1.00 & - & - & - & - & - & - & - \\
\hline 112 & -0.9701 & 0.0137 & 5.7 & -59.32 & 1.61 & - & - & - & - & - & - & - \\
\hline 113 & -0.9703 & 0.0141 & 0.1 & -56.58 & 2.00 & - & - & - & - & - & - & - \\
\hline 114 & -0.9726 & 0.0516 & 0.03 & -39.74 & 0.94 & - & - & - & - & - & - & - \\
\hline 115 & -0.9738 & 0.0125 & 0.5 & -51.32 & 0.65 & - & - & - & - & - & - & - \\
\hline 116 & -0.9746 & 0.0136 & 0.8 & -60.16 & 2.13 & - & - & - & - & - & - & - \\
\hline 117 & -0.9815 & -0.0441 & 0.8 & -60.16 & 0.64 & - & - & - & - & - & - & - \\
\hline 118 & -0.9816 & -0.0441 & 0.7 & -60.16 & 0.62 & - & - & - & - & - & - & - \\
\hline 119 & -0.9849 & -0.0415 & 0.2 & -58.48 & 1.12 & - & - & - & - & - & - & - \\
\hline
\end{tabular}

$\begin{array}{llllll}120 & -1.1899 & -0.3140 & 0.5 & -50.90 & 0.49 \\ 121 & -1.1933 & -0.3141 & 7.8 & -50.90 & 0.60 \\ 122 & -1.2056 & -0.2854 & 0.4 & -51.32 & 0.51\end{array}$

$\begin{array}{lll}123 & -1.4045 & 0.1428 \\ 124 & -1.4384 & 0.1261 \\ 125 & -1.4432 & 0.1242 \\ 126 & -1.4908 & 0.1022 \\ 127 & -1.5437 & 0.0778 \\ 128 & -1.7615 & 0.1491 \\ 129 & -1.7825 & 0.1442\end{array}$

$\begin{array}{lll}5.5 & -51.11 & 0.48 \\ 1.8 & -46.90 & 0.58 \\ 5.6 & -44.16 & 0.66 \\ 0.3 & -48.79 & 1.64 \\ 0.3 & -51.11 & 0.67 \\ 2.1 & -53.63 & 0.69 \\ 0.2 & -54.27 & 0.59\end{array}$

Cluster " $e$ "

$$
\begin{array}{ccccccc}
- & - & - & - & - & - & - \\
2.1 \pm 0.1 & +52 \pm 1 & 1.1_{-0.1}^{+0.5} & 10.5_{-0.4}^{+0.2} & - & - & \mathbf{6 2}_{-41}^{+15} \\
- & - & - & - & - & - & -
\end{array}
$$

Cluster " $b$ "

$$
\begin{array}{ccccccc}
8.0 \pm 1.5 & +37 \pm 7 & 1.6_{-0.4}^{+0.7} & 9.5_{-1.8}^{+0.7} & - & - & 90_{-11}^{+11} \\
- & - & - & - & - & - & - \\
- & - & - & - & - & - & - \\
- & - & - & - & - & - & - \\
- & - & - & - & - & - & - \\
6.4 \pm 1.0 & -55 \pm 10 & 1.0_{-0.8}^{+0.7} & 10.3_{-2.2}^{+0.7} & - & - & 80_{-7}^{+8} \\
- & - & - & - & - & - & -
\end{array}
$$

\begin{tabular}{|c|c|c|c|c|c|c|c|c|c|c|c|c|}
\hline 130 & -2.0157 & -0.2032 & 0.6 & -51.11 & 0.76 & - & - & - & - & - & - & - \\
\hline 131 & -2.0164 & -0.2007 & 26.1 & -49.63 & 1.39 & $2.6 \pm 2.0$ & $+86 \pm 3$ & $2.5_{-1.0}^{+0.1}$ & $10.6_{-0.1}^{+0.4}$ & - & - & $63_{-55}^{+3}$ \\
\hline 132 & -2.0169 & -0.2007 & 27.1 & -48.37 & 1.17 & $2.6 \pm 0.7$ & $-87 \pm 8$ & $2.4_{-1.1}^{+0.0}$ & $10.6_{-0.1}^{-0.1}$ & - & - & $63_{-42}^{+25}$ \\
\hline 133 & -2.0177 & -0.2009 & 0.6 & -51.53 & 1.26 & - & - & - & - & - & - & - \\
\hline 134 & -2.0178 & -0.2009 & 7.6 & -49.00 & 1.45 & $5.0 \pm 1.5$ & $+86 \pm 9$ & $2.4_{-1.3}^{+0.1}$ & $10.6_{-4.5}^{+0.4}$ & - & - & $69_{-39}^{+9}$ \\
\hline 135 & -2.0179 & -0.2005 & 0.8 & -45.00 & 1.04 & - & - & - & - & - & - & - \\
\hline 136 & -2.0187 & -0.1970 & 0.2 & -41.00 & 0.79 & - & - & - & - & - & - & - \\
\hline 137 & -2.0449 & -0.1574 & 7.2 & -45.21 & 0.50 & $3.3 \pm 0.5$ & $-24 \pm 1$ & $1.0_{-0.8}^{+0.5}$ & $10.2_{-1.9}^{+0.7}$ & - & - & $72_{-6}^{+15}$ \\
\hline 138 & -2.0645 & 0.2090 & 4.6 & -41.84 & 0.58 & - & - & - & - & - & - & - \\
\hline 139 & -2.0706 & 0.1342 & 0.6 & -41.21 & 0.61 & - & - & - & - & - & - & - \\
\hline 140 & -2.0724 & 0.1375 & 13.2 & -36.79 & 0.89 & $1.4 \pm 0.1$ & $+80 \pm 5$ & $1.4_{-0.2}^{+0.1}$ & $9.5_{-0.3}^{+0.2}$ & - & - & $69_{-32}^{+12}$ \\
\hline 141 & -2.0853 & -0.2909 & 0.5 & -50.48 & 1.83 & - & - & - & - & - & - & - \\
\hline 142 & -2.0873 & -0.3049 & 5.5 & -47.32 & 0.62 & - & - & - & - & - & - & - \\
\hline 143 & -2.0875 & -0.3067 & 0.1 & -46.26 & 0.95 & - & - & - & - & - & - & - \\
\hline 144 & -2.1038 & 0.2021 & 1.0 & -43.95 & 0.96 & - & - & - & - & - & - & - \\
\hline 145 & -2.1099 & 0.1926 & 0.4 & -42.68 & 0.59 & - & - & - & - & - & - & - \\
\hline 146 & -2.0152 & -0.2030 & 0.3 & -51.95 & 1.28 & - & - & - & - & - & - & - \\
\hline 147 & -2.1208 & -0.2892 & 3.8 & -47.74 & 0.84 & - & - & - & - & - & - & - \\
\hline 148 & -2.1228 & -0.2879 & 0.9 & -47.11 & 0.66 & - & - & - & - & - & - & - \\
\hline
\end{tabular}

Cluster " $c$ " 